\title{
Frustrated Magnetism in Mott Insulating $\left(\mathrm{V}_{1-x} \mathrm{Cr}_{x}\right)_{2} \mathrm{O}_{3}$
}

\author{
J. C. Leiner, ${ }^{1,2,}{ }^{*}$ H. O. Jeschke, ${ }^{3}$ R. Valentî, ${ }^{4}$ S. Zhang, ${ }^{5}$ A. T. Savici, ${ }^{2}$ J. Y. Y. Lin, ${ }^{2}$ M. B. Stone,${ }^{2}$ \\ M. D. Lumsden, ${ }^{2}$ Jiawang Hong, ${ }^{6,7}$ O. Delaire, ${ }^{8,7}$ Wei Bao, ${ }^{9}$ and C. L. Broholm ${ }^{5,2}$ \\ ${ }^{1}$ Center for Correlated Electron Systems, Institute for Basic Science (IBS), Seoul 08826, Korea \\ and Department of Physics and Astronomy, Seoul National University, Seoul 08826, Korea \\ ${ }^{2}$ Neutron Scattering Division, Oak Ridge National Laboratory, Oak Ridge, Tennessee 37831, USA \\ ${ }^{3}$ Research Institute for Interdisciplinary Science, Okayama University, Okayama 700-8530, Japan \\ ${ }^{4}$ Institut für Theoretische Physik, Goethe-Universität Frankfurt, Max-von-Laue-Straße 1, \\ 60438 Frankfurt am Main, Germany \\ ${ }^{5}$ Institute for Quantum Matter and Department of Physics and Astronomy, The Johns Hopkins University, \\ Baltimore, Maryland 21218, USA \\ ${ }^{6}$ School of Aerospace Engineering, and Institute of Advanced Structure Technology, \\ Beijing Institute of Technology, Beijing 100081, China \\ ${ }^{7}$ Materials Science and Technology Division, Oak Ridge National Laboratory, Oak Ridge, \\ Tennessee 37831, USA \\ ${ }^{8}$ Department of Mechanical Engineering and Materials Science and Department of Physics, \\ Duke University, Durham, North Carolina 27708, USA \\ ${ }^{9}$ Department of Physics, Renmin University of China, Beijing 100872, China
}

(Received 23 April 2018; revised manuscript received 14 October 2018; published 21 February 2019)

\begin{abstract}
$\mathrm{V}_{2} \mathrm{O}_{3}$ famously features all four combinations of paramagnetic versus antiferromagnetic and metallic versus insulating states of matter in response to percent-level doping, pressure in the GPa range, and temperature below $300 \mathrm{~K}$. Using time-of-flight neutron spectroscopy combined with density functional theory calculations of magnetic interactions, we have mapped and analyzed the inelastic magnetic neutron scattering cross section over a wide range of energy and momentum transfer in the chromium-stabilized antiferromagnetic and paramagnetic insulating phases. Our results reveal an important magnetic frustration and degeneracy of the paramagnetic insulating phase which is relieved by the rhombohedral-to-monoclinic transition at $T_{N}=185 \mathrm{~K}$. This leads to the recognition that magnetic frustration is an inherent property of the paramagnetic phase in $\left(\mathrm{V}_{1-x} \mathrm{Cr}_{x}\right)_{2} \mathrm{O}_{3}$ and plays a key role in suppressing the magnetic long-rangeordering temperature and exposing a large phase space for the paramagnetic Mott metal-insulator transition to occur.
\end{abstract}

DOI: 10.1103/PhysRevX.9.011035

Subject Areas: Magnetism,

Strongly Correlated Materials

\section{INTRODUCTION}

Metal-to-insulator transitions (MIT) come in many guises, but for transition-metal oxides they generally involve magnetic or structural symmetry breaking. $\mathrm{V}_{2} \mathrm{O}_{3}$, however, offers a singular counterexample. As a function of chromium doping, $\left(\mathrm{V}_{1-x} \mathrm{Cr}_{x}\right)_{2} \mathrm{O}_{3}$ undergoes a paramagnetic metal-to-insulator transition that is accompanied by a volume expansion but no global magnetostructural

\footnotetext{
* Corresponding author. jleiner@alumni.nd.edu

Published by the American Physical Society under the terms of the Creative Commons Attribution 4.0 International license. Further distribution of this work must maintain attribution to the author(s) and the published article's title, journal citation, and DOI.
}

symmetry breaking [Fig. 1(a)] [1-3]. In this paper, we identify magnetic frustration in paramagnetic insulating (PI) $\left(\mathrm{V}_{1-x} \mathrm{Cr}_{x}\right)_{2} \mathrm{O}_{3}$ as a rare characteristic that contributes to expose the available paramagnetic phase space for the Mott metal-insulator transition [4] by suppressing longrange magnetic order. Furthermore, we show that, in contrast to the PI phase, the magnetic interactions of the monoclinic antiferromagnetic insulating (AFI) phase are all simultaneously satisfied in the ordered state. The magnetostructural transition from the PI to AFI phase [Fig. 1(a)] thus lifts degeneracies associated with frustrated interactions in a spin-Peierls-like transition [5] as in other frustrated magnets such as $\mathrm{ZnCr}_{2} \mathrm{O}_{4}[6]$ and $\mathrm{ZnV}_{2} \mathrm{O}_{4}[7,8]$.

Our conclusions are based on a careful examination of magnetic interactions in $\left(\mathrm{V}_{0.96} \mathrm{Cr}_{0.04}\right)_{2} \mathrm{O}_{3}$ through inelastic neutron scattering (INS) and density-functional theory (DFT) total energy calculations. The low- $T$ monoclinic antiferromagnetic (AFM) insulator has conventional 

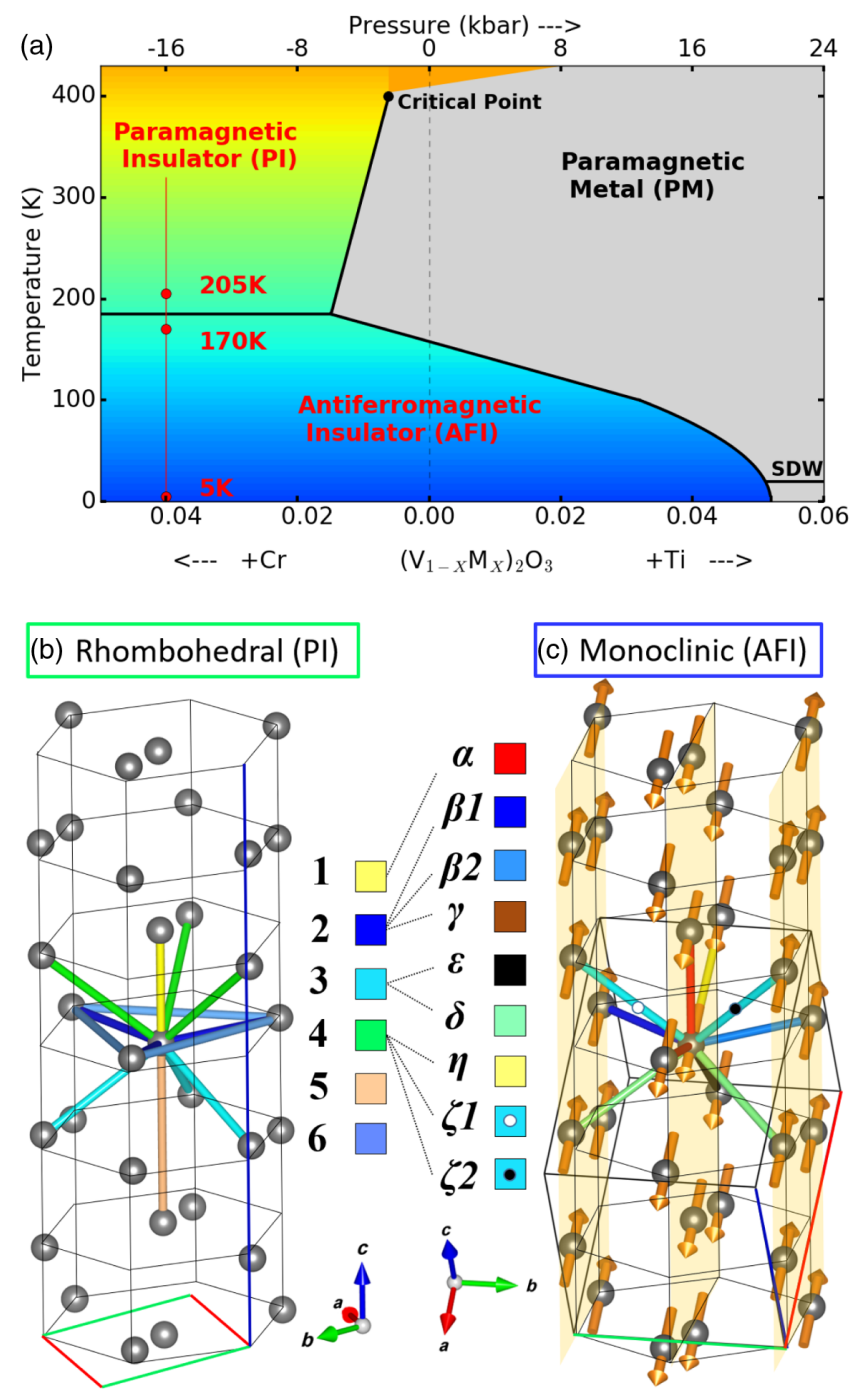

FIG. 1. (a) $\mathrm{V}_{2} \mathrm{O}_{3}$ phase diagram showing the extent of the AFI, PI, and PM phases [14] as a function of both chemical substitution (bottom axis) and the equivalent applied pressure (top axis). (b) The rhombohedral structure of the PI phase, with the color-labeled exchange interactions (1-6) determined in the paper. Black hexagons outline the pseudohexagonal (corundum) unit cell. (c) The distorted crystal structure (monoclinic unit cell shown) and magnetic structure in the AFI phase [15], where the magnetic moments are ferromagnetically aligned in monoclinic $a+c$ planes, which are then stacked antiferromagnetically along the monoclinic $b$ axis. Greek indices indicate the updated nearestneighbor bond configuration resulting from the monoclinic distortion. Oxygen atoms are omitted for clarity.

spin-wave excitations that allow us to determine the nonfrustrated exchange Hamiltonian by measuring and analyzing the spin-wave dispersion relation. The agreement between the experimentally extracted Hamiltonian and the one calculated by DFT establishes DFT as a valid method to determine the dominant exchange constants in $\mathrm{V}_{2} \mathrm{O}_{3}$. We then perform DFT calculations to discover the key exchange constants in the PI phase and show, using a Gaussian approximation, that these are consistent with measured diffuse magnetic neutron scattering. The PI phase is a strongly frustrated quasi-two-dimensional honeycomb antiferromagnet with a nominal critical temperature below $10 \mathrm{~K}$. That phase transition is, however, preempted by a spin-Peierls-like magnetostructural transition at $185 \mathrm{~K}$.

Our observations suggest that the large region of paramagnetism in the phase diagram of $\mathrm{V}_{2} \mathrm{O}_{3}$ is made possible by magnetic frustration which deeply suppresses magnetic order in the Mott insulator. This reinforces the importance of exploring metallization of frustrated magnets through doping $[9,10]$ and pressure [11-13].

\section{A. Summary of prior work on $\mathbf{V}_{2} \mathbf{O}_{3}$}

While our work reveals a previously unappreciated aspect of $\mathrm{V}_{2} \mathrm{O}_{3}$, it is by no means the first effort to understand spin interactions in this material [14,16-23]. In a comprehensive work in 1978, Castellani et al. [24-26] invoked the formation of vanadium dimers and $e_{g}$ orbital order to explain the measured antiferromagnetic structure [15] in the AFI phase. Many other theoretical studies of $\mathrm{V}_{2} \mathrm{O}_{3}$ used simpler one-orbital models which neglect the orbital degrees of freedom and assume the same kind of AFM correlations for all phases of $\mathrm{V}_{2} \mathrm{O}_{3}$ [27]. In 1993, a different kind of AFM order was found in the metallic phase of $\mathrm{V}_{2-y} \mathrm{O}_{3}[28,29]$ stabilized to low temperatures through vanadium deficiency. It was further shown through INS that the phase transition to the AFI phase, from either the metallic or the insulating paramagnetic phases, abruptly shifts the critical magnetic wave vector from $(10 \ell)$ to $\left(\frac{1}{2} \frac{1}{2} 0\right)$ $[30,31]$. This is inconsistent with predictions from oneorbital theories, and indicates the transition is not a conventional order-to-disorder type of magnetic transition. This behavior seemed to be naturally explained within the coupled spin-orbital model of Rice [32], as an orbital ordering transition with accompanying antiferromagnetism. The symmetric SU(4) spin-orbital model has since attracted considerable theoretical interest [33-35].

Subsequent resonant $\mathrm{x}$-ray scattering experiments discovered a new type of Bragg peak [36], which was explained as the order parameter of the $e_{g}$ orbital ordering $[37,38]$. Polarization-dependent $\mathrm{x}$-ray absorption spectroscopy measurements [39] reached the unexpected conclusion that there is an $S=1$ state at each $\mathrm{V}$ site, with dominant orbital occupation in $e_{g}^{\pi}$ and a small admixture of the $a_{1 g}$ orbital. This was supported by LDA $+U$ [40] and LDA + DMFT [41,42] calculations with selected values for the Hubbard and the Hund's coupling energies. The calculated magnetic structures in LDA $+U$ [40], determined without orbital degeneracy or orbital ordering being necessary, were found to be consistent with experiments. Alternatively, an $S=1$ model with orbital degeneracy was suggested [43-45] in which stable magnetic and orbital structures were systematically analyzed and anomalous features of the AFI transition were qualitatively explained. In this picture, the phase transition from PI to AFI was 
interpreted as being fundamentally a structural and orbital occupational ordering transition [46] that brings the spin system deeply into a long-range-ordered state without the usual critical regime associated with a growing spin correlation length.

In recent years, angle-resolved photoemission spectroscopy measurements [47] and fully charge self-consistent LDA + DMFT calculations $[1,4,48,49]$ have indicated a much weaker orbital polarization than initially suggested $[41,42]$, which may influence the nature of the paramagnetic metal-to-insulator transition. Furthermore, the authors of Ref. [4] found that changes in the $\mathrm{V}_{2} \mathrm{O}_{3}$ paramagnetic phase diagram are driven by defect-induced local symmetry breakings resulting from different couplings of $\mathrm{Cr}$ and $\mathrm{Ti}$ dopants to the host system. These results suggest that local distortions which lift orbital degeneracy play an important role in the description of the insulating phase in Cr-doped $\mathrm{V}_{2} \mathrm{O}_{3}$.

\section{B. Outline}

In view of ongoing efforts to understand the insulating phases in $\mathrm{V}_{2} \mathrm{O}_{3}$, it is important to provide an experimental anchor with reliable values of magnetic exchange interactions that limit the parameter space of $S=1$ theories. This is possible in the AFI phase by measuring spin-wave dispersion relations and comparing those to linear spinwave theory (LSWT). The acoustic branch of spin waves was previously measured using INS near the magnetic zone center for $\mathrm{V}_{2} \mathrm{O}_{3}$ and $\left(\mathrm{V}_{0.96} \mathrm{Cr}_{0.04}\right)_{2} \mathrm{O}_{3}$ in the AFI phase [50-52]. The limited range of those data, however, is insufficient to determine the many distinct exchange interactions of the monoclinic phase [52].

Here we report INS measurements of both acoustic and optic branches of spin waves in the AFI phase and incoherent magnetic excitations in the PI phase throughout the Brillouin zone for $\left(\mathrm{V}_{0.96} \mathrm{Cr}_{0.04}\right)_{2} \mathrm{O}_{3}$. At room temperature $\mathrm{V}_{2} \mathrm{O}_{3}$ has the trigonal (corundum) structure with space group $R \overline{3} c$ (no. 167) while the space group of the low-temperature monoclinic structure is $I 2 / \mathrm{a}$ (no. 15) with 8 vanadium ions per unit cell. Our new measurements and new $a b$ initio DFT calculation methods for $\left(\mathrm{V}_{0.96} \mathrm{Cr}_{0.04}\right)_{2} \mathrm{O}_{3}$ allow for the accurate determination of all magnetic exchange interactions in the monoclinic longrange-ordered AFM state of vanadium sesquioxide. We find moderate ferromagnetic nearest-neighbor and dominant AFM next-nearest-neighbor interactions in an unfrustrated configuration along the zigzag $\mathrm{V}$ chains in the AFI phase [Fig. 1(c)]. Having established the effectiveness of our DFT calculation methods in the AFI phase, we apply them to determine an interacting spin Hamiltonian for the PI phase. A Gaussian approximation applied to this model accounts in detail for our measurements of the short-range spin correlations in the PI phase. This leads to the conclusion that the lack of spin order for temperatures down to $185 \mathrm{~K}$ in the PI phase is a consequence of frustrated interactions on the puckered honeycomb lattices that make up the corundum structure.

\section{EXPERIMENTAL METHODS}

Single-crystal samples of $\left(\mathrm{V}_{0.96} \mathrm{Cr}_{0.04}\right)_{2} \mathrm{O}_{3}\left(T_{N}=185 \mathrm{~K}\right)$ were grown using a skull melter [53]. To increase the sensitivity of our experiment, four single crystals were coaligned for a total mass of $17 \mathrm{~g}$. In the PI phase at $T=296 \mathrm{~K}$, the pseudohexagonal lattice constants are $a=$ $b=4.94 \AA$ and $c=14.01 \AA$. In the AFI phase at $T=77 \mathrm{~K}$, the monoclinic lattice parameters are $a=7.28 \AA, b=$ $4.99 \AA, c=5.54 \AA\left(\alpha, \beta, \gamma=\left[90^{\circ}, 96.75^{\circ}, 90^{\circ}\right]\right)$, consistent with previous findings $[17,54,55]$.

We use both the primitive monoclinic unit cell and the pseudohexagonal unit cell to label reciprocal space (Fig. 1). The AFM wave vector $\left(\begin{array}{lll}\frac{1}{2} & \frac{1}{2} & 0\end{array}\right)_{H}$ in the pseudohexagonal unit cell becomes $\left(\begin{array}{lll}0 & 1 & 0\end{array}\right)_{M}$ in the monoclinic unit cell. The conversion between pseudohexagonal and monoclinic coordinates is approximately given by

$$
\left(\begin{array}{l}
H_{M} \\
K_{M} \\
L_{M}
\end{array}\right)=\left(\begin{array}{ccc}
\frac{2}{3} & -\frac{2}{3} & -\frac{1}{3} \\
1 & 1 & 0 \\
\frac{1}{3} & -\frac{1}{3} & \frac{1}{3}
\end{array}\right)\left(\begin{array}{l}
H_{H} \\
K_{H} \\
L_{H}
\end{array}\right) .
$$

Time-of-flight INS measurements were performed using the SEQUOIA fine-resolution Fermi-chopper spectrometer at the Spallation Neutron Source at ORNL [56,57]. (Initial measurements were performed at the MARI multidetector chopper spectrometer at the pulsed spallation source at ISIS, UK.) With this modern pulsed-neutron spectrometer, we were able to fully map the excitation spectrum of $\left(\mathrm{V}_{0.96} \mathrm{Cr}_{0.04}\right)_{2} \mathrm{O}_{3}$ at three key temperatures indicated in Fig. 1(a). The crystal array was aligned so that the $(-L K L)_{M}$ plane was horizontal. The crystal assembly was rotated through $180^{\circ}$ in $2^{\circ}$ steps about the vertical $(H 0 H / 2)_{M}$ direction to access a volume of momentum space during the experiment. To balance the energy resolution with $\mathbf{Q}$-space coverage, incident energies of 50 and $100 \mathrm{meV}$ were used to collect data at $(5 \mathrm{~K}, 170 \mathrm{~K}, 205 \mathrm{~K})$ and $(5 \mathrm{~K}, 205 \mathrm{~K}, 320 \mathrm{~K})$, respectively.

The magnetic neutron scattering cross section for momentum transfer $\mathbf{Q}=\mathbf{k}_{i}-\mathbf{k}_{f}$ and energy transfer $\hbar \omega=E_{i}-E_{f}$ is given by

$$
\begin{aligned}
\frac{d^{2} \sigma}{d \Omega d E_{f}}= & N \frac{k_{f}}{k_{i}} r_{0}^{2} e^{-2 W(Q)}\left|\frac{g}{2} f(\mathbf{Q})\right|^{2} \\
& \times \sum_{\alpha \beta}\left(\delta_{\alpha \beta}-\hat{Q}_{\alpha} \hat{Q}_{\beta}\right) \mathcal{S}^{\alpha \beta}(\mathbf{Q}, \omega),
\end{aligned}
$$

where $N$ is the number of hexagonal unit cells, $r_{0}=$ $0.539 \times 10^{-12} \mathrm{~cm}, f(\mathbf{Q})$ is the magnetic form factor for the $\mathrm{V}^{3+}$ ion, and $e^{-2 W(Q)}$ is the Debye-Waller factor. The dynamic spin correlation function (or dynamical structure factor) is given by 


$$
\begin{aligned}
\mathcal{S}^{\alpha \beta}(\mathbf{Q}, \omega)= & \frac{1}{2 \pi \hbar} \int d t e^{-i \omega t} \frac{1}{N} \sum_{\mathbf{R}, \mathbf{R}^{\prime}} e^{i \mathbf{Q} \cdot\left(\mathbf{R}-\mathbf{R}^{\prime}\right)} \\
& \times\left\langle S_{\mathbf{R}}^{\alpha}(0) S_{\mathbf{R}^{\prime}}^{\beta}(t)\right\rangle .
\end{aligned}
$$

To enhance statistical quality, we employed the rotational symmetry operations of the PI phase to project the data into an irreducible wedge. In addition, the MANTID software suite [58] was used to subtract an incoherent background (see Appendix A 1). The intensity data were normalized as described in Appendix A 2 so that we report the measured scattering cross section in absolute units as

$$
\mathcal{I}(\mathbf{Q}, \omega)=\frac{k_{i}}{k_{f}} \frac{1}{N} \frac{d^{2} \sigma}{d \Omega d E_{f}}
$$

\section{RESULTS}

\section{A. Overview of $\mathcal{S}(\mathbf{Q})$}

In Fig. 2, we show the momentum dependence of the spin-correlation function $\mathcal{S}(\mathbf{Q})$ averaged over an energy range 5-10 meV. The data were acquired with an incident energy $E_{i}=50 \mathrm{meV}$ at temperatures 205, 170, and $5 \mathrm{~K}$. Figures 2(a), 2(d), and 2(g) show $\mathcal{S}(\mathbf{Q})$ for the pseudohexagonal basal plane $\mathbf{Q}=(H K 0)_{H}$. It is clear from the lowtemperature data [Figs. 2(g) and 2(i)] that there are three monoclinic domains in the hexagonal basal plane (each $60^{\circ}$ apart). Their volume fractions of $56 \%, 26 \%$, and $18 \%$, respectively, were determined from Fig. $2(\mathrm{~g})$ and duly incorporated into the forthcoming simulations. The unequal domain population can arise from symmetry breaking associated with the sample shape and the strain imposed by the sample mounting provisions.

At $T=205 \mathrm{~K}$ [Figs. 2(a)-2(c)], we observe diffuse scattering that we associate with short-range correlated paramagnetic spin fluctuations. Consistent with rhombohedral symmetry, the sixfold structure of magnetic neutron scattering in the pseudohexagonal basal plane $L_{H}=0$ [Figs. 2(a), 2(d), and 2(g)] becomes a threefold structure for $L_{H} \neq 3 \times$ (integer) [Figs. 2(c), 2(f), 2(i)]. In these new data we see for the first time the direction, $\left(\begin{array}{lll}H & 0 & 0\end{array}\right)_{M}$ [indicated by the green arrow no. 3 in Fig. 2(h)], along which coherent spin waves abruptly "melt" into a broad paramagnon excitation at wave vectors located between the acoustic spin-wave branches.

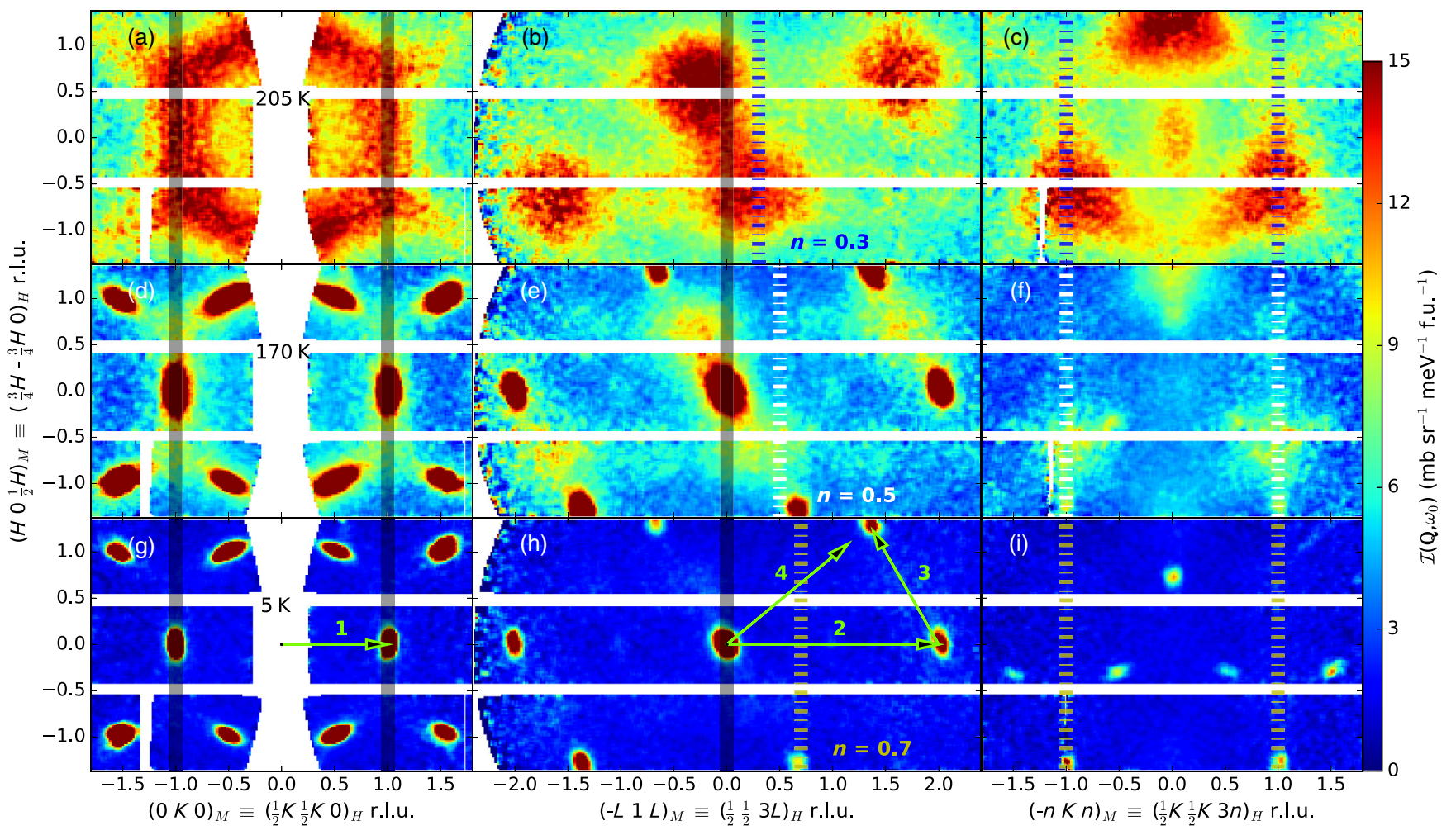

FIG. 2. Constant energy slices of INS data $\left(E_{i}=50 \mathrm{meV}\right)$ for $\left(\mathrm{V}_{0.96} \mathrm{Cr}_{0.04}\right)_{2} \mathrm{O}_{3}$; energy transfer averaged from $5<\hbar \omega_{0}<10 \mathrm{meV}$. Each horizontal row shows $\mathcal{I}\left(\mathbf{Q}, \omega_{0}\right)$ for the three temperatures marked along the phase diagram in Fig. 1. The left-hand column, (a), (d), and $(\mathrm{g})$, shows $\mathcal{I}\left(\mathbf{Q}, \omega_{0}\right)$ in the pseudohexagonal basal plane at the origin $(L=0)$. The center column, (b), (e), and (h), covers a plane perpendicular to that of the left-hand column overlapping at the light gray solid lines. The right-hand column, (c), (f), and (i), shows pseudohexagonal planes for $L_{H}=3 n$, where $n=0.3,0.5$, and 0.7 for frames (c), (f), and (i), respectively, as indicated in the center column by the colored dashed lines. Green arrows indicate the four high-symmetry directions used in Fig. 3. 
A dramatic change in the character of the magnetic excitations across the first-order AFI to PI phase transition is also apparent in the $\mathbf{Q}-E$ slices of Fig. 3(d). [Note that the qualitative features of Fig. 3(d) persist up to at least $320 \mathrm{~K}$, as shown in Appendix B 1, Fig. 9(d)]. The sharp excitations near $80 \mathrm{meV}$ energy transfer along the $\left(\begin{array}{lll}-L & 1 L\end{array}\right)_{M}$

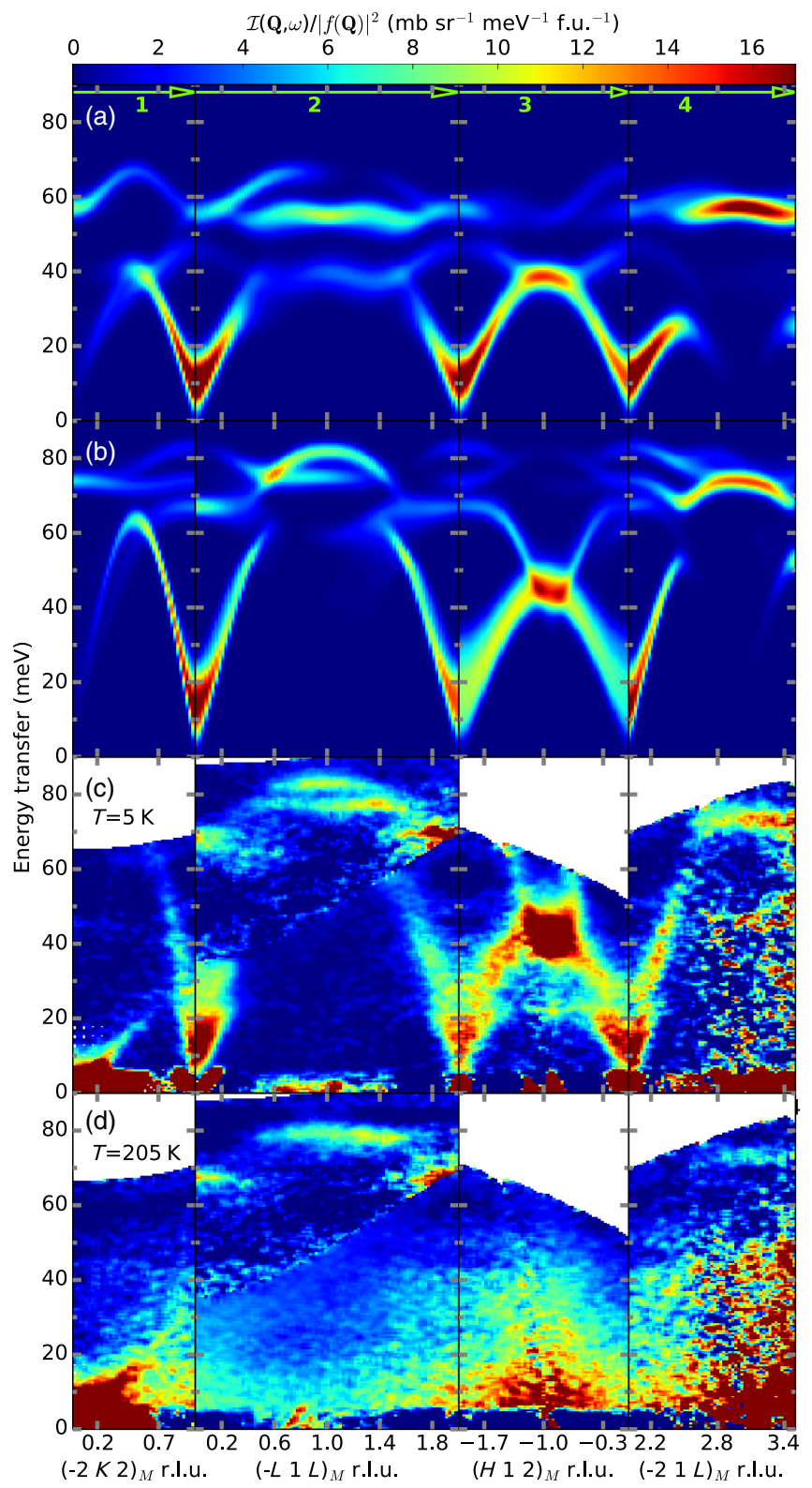

FIG. 3. Neutron scattering intensity associated with spin waves along four high-symmetry directions marked in Fig. 2 using (a) DFT calculated exchange constants for $\left(\mathrm{V}_{0.96} \mathrm{Cr}_{0.04}\right)_{2} \mathrm{O}_{3}$ and (b) exchange constants obtained from fits to the data. (c),(d) INS cross section measured at 5 and $205 \mathrm{~K}\left(E_{i}=100 \mathrm{meV}\right)$. Data from multiple Brillouin zones were assembled to cover the largest possible range of energy transfer. The data were divided by the squared magnetic form factor for presentation. Also, an incoherent background determined by azimuthal averaging of the same data was subtracted (Appendix A 1). and $\left(\begin{array}{llll}-2 & 1 & L\end{array}\right)_{M}$ directions that survive to $320 \mathrm{~K}$ would appear to be phonons.

\section{B. Exchange interactions in the AFI phase}

\section{Neutron scattering}

Our comprehensive INS dataset acquired for $E_{i}=$ $100 \mathrm{meV}$ provides access to both acoustic and optic spinwave excitations from the AFI phase [Fig. 3(c)]. Along all high-symmetry directions, sharp dispersive ridges of magnetic scattering are observed indicative of coherent spin-wave-like excitations in the AFI phase. These can be described as the normal modes of excitation from the ordered state of an antiferromagnet described by a spin Hamiltonian of the form

$$
\mathcal{H}_{\text {mag }}=\sum_{i, j} J_{i j} \mathbf{S}_{i} \cdot \mathbf{S}_{j}+\sum_{i} D\left(S_{i}^{z}\right)^{2}
$$

Here $J_{i j}$ are exchange constants for interaction between spins $i$ and $j(S=1)$ that we approximate as Heisenberglike (isotropic in spin space). The symmetry inequivalent exchange interactions in the PI and AFI phases are defined in Figs. 1(b) and 1(c). A derivation of the corresponding spin-wave dispersion relation in the monoclinic AFI phase was first presented in Ref. [52]. For simplicity, the measured $4.8 \mathrm{meV}$ energy gap in the spin-wave excitation spectrum is wholly attributed to a uniaxial single ion anisotropy term $D=0.13 \mathrm{meV}$, even though exchange anisotropy must also be present $[50,52]$.

For a given set of exchange constants, the inelastic neutron scattering cross section associated with spin-wave excitations from the AFI state was calculated with linear spin-wave theory using the SPINW program [59] and then convoluted with the energy-dependent instrumental resolution function [56,57]. By comparing the measured and calculated spectra along the four high-symmetry directions [Figs. 2(g) and 2(h)], we inferred the best-fit exchange parameters listed in column 5 of Table I. [The error bars given are an upper bound expressing the range of each exchange parameter value that allows for an acceptable overall fit of the INS data in Fig. 3(c).] The INS that we associate with spin waves [Fig. 3(c)] is well accounted for by the model [Fig. 3(b)], which gives confidence that the corresponding exchange Hamiltonian describes magnetic interactions within the AFI phase.

\section{Density-functional theory}

DFT electronic structure calculations for the monoclinic AFI phase were performed using the $T=15 \mathrm{~K}$ structure of Rozier et al. [60] with the full potential local orbital (FPLO) basis set [61] and the generalized gradient approximation (GGA) and GGA $+U$ functional [62]. We obtained up to twelfth nearest-neighbor exchange constants by mapping total energy differences of various magnetic configurations 
TABLE I. Magnetic exchange constants (in units of meV) for the AFI phase in $\left(\mathrm{V}_{0.96} \mathrm{Cr}_{0.04}\right)_{2} \mathrm{O}_{3}$, obtained from both DFT calculations and direct fitting of the neutron data as described in the main text. Column 6 gives the sign of the indicated correlation $\left\langle\mathbf{S}_{i} \cdot \mathbf{S}_{j}\right\rangle$ for the AFI structure [Fig. 1(c)]. The consistent negative sign of the product between columns 5 and 6 indicates an unfrustrated magnet.

\begin{tabular}{lcrcrc}
\hline \hline $\begin{array}{l}\text { Distance } \\
(\AA)\end{array}$ & $\begin{array}{c}J_{i} \\
(S=1)\end{array}$ & \multicolumn{1}{c}{$\begin{array}{c}\mathrm{DFT} \\
\mathrm{V}_{2} \mathrm{O}_{3}\end{array}$} & $\begin{array}{c}\mathrm{DFT} \\
\left(\mathrm{V}_{0.96} \mathrm{Cr}_{0.04}\right)_{2} \mathrm{O}_{3}\end{array}$ & $\begin{array}{r}\text { Data } \\
(\mathrm{Fit})\end{array}$ & $\begin{array}{l}\text { Sign of } \\
\left\langle\mathbf{S}_{i} \cdot \mathbf{S}_{j}\right\rangle\end{array}$ \\
\hline 2.75904 & $J_{\alpha}$ & $2.8(3)$ & $-1(2)$ & $-6.0(2)$ & + \\
2.83083 & $J_{\beta 1}$ & $28.7(3)$ & $25(2)$ & $27.7(2)$ & - \\
2.91789 & $J_{\beta 2}$ & $12.4(3)$ & $9(2)$ & $7.7(2)$ & - \\
2.98538 & $J_{\gamma}$ & $-2.3(3)$ & $3(2)$ & $0.0(2)$ & + \\
3.43336 & $J_{\epsilon}$ & $-3.9(5)$ & $-9(3)$ & $2.0(2)$ & - \\
3.45420 & $J_{\delta}$ & $3.5(3)$ & $4(2)$ & $1.1(2)$ & - \\
3.63334 & $J_{\eta}$ & $0.6(3)$ & $1(2)$ & $-2.0(2)$ & + \\
3.70177 & $J_{\zeta 1}$ & $2.5(2)$ & $1(1)$ & $7.1(2)$ & - \\
3.76876 & $J_{\zeta 2}$ & $-0.3(2)$ & $-1(1)$ & $7.1(2)$ & - \\
4.22293 & $J_{\theta}$ & $0.8(3)$ & $-4(2)$ & 0 & + \\
4.97765 & $J_{l}$ & $0.4(2)$ & $3(1)$ & 0 & - \\
5.00240 & $J_{\kappa}$ & $1.7(2)$ & $-1(1)$ & 0 & + \\
\hline \hline
\end{tabular}

to the Heisenberg model [63]. Table I, third column, displays the calculated exchange constants for pure $\mathrm{V}_{2} \mathrm{O}_{3}$ with $U=3 \mathrm{eV}$ (see also Appendix B 1). While these values were obtained from total energy calculations, where all contributions of crystal-field splittings and orbital hybridization paths-as dictated by the details of the crystal structureare integrally taken into account but difficult to extract individually, perturbation expansion considerations indicate that these exchange interactions strongly depend on crystalfield splittings and hoppings between $a_{1 g}$ and $e_{g}$ states [64] as previously found for other multiorbital systems $[65,66]$.

The experimental determination of the AFI phase exchange constants (Table I, fifth column) conveniently serves to benchmark the $a b$ initio calculations of exchange interactions. While the agreement for the dominant exchange constants (third and fifth columns in Table I) is rather good, however, we found that to achieve quantitative agreement with the experimental $J$ values, in particular the sign of $J_{\alpha}$, accounting for the $4 \% \mathrm{Cr}(S=3 / 2)$ doping in the sample was necessary. Introducing $\mathrm{Cr}$ doping into the DFT calculations is quite challenging and leads to significantly higher statistical error ranges. Two complementary approaches were employed: (1) replacing one $\mathrm{V}^{3+}$ ion by a $\mathrm{Cr}^{3+}$ ion in a large $\mathrm{V}_{2} \mathrm{O}_{3}$ supercell where the different bonding environment of $\mathrm{Cr}^{3+}$ is explicitly taken into account and (2) increasing the average nuclear charge from $Z=23$ (pure $\mathrm{V}_{2} \mathrm{O}_{3}$ ) to $Z=23.04\left[\left(\mathrm{~V}_{0.96} \mathrm{Cr}_{0.04}\right)_{2} \mathrm{O}_{3}\right]$ and using the virtual crystal approximation. Both of these approaches can deliver reasonable indications concerning the effect of $\mathrm{Cr}$ doping. We found that in both cases the $\mathrm{Cr}$ doping indeed introduces a tendency towards FM $J_{\alpha}<0$ in better agreement with the experimental data. As previously noted, local changes in the crystal structure induced by $\mathrm{Cr}$ doping play an important role in the MIT [4]. However, the resulting exchange coupling constants from these calculations do not show drastic changes upon $\mathrm{Cr}$ doping. An explanation for this is that the magnetic exchange constants are roughly $J \propto-t^{2} /(U+\Delta)$, where $U \gg \Delta$ is the dominant scale and therefore they are not expected to be highly sensitive to effects related to changes of the order of the crystal field $\Delta$. The DFT calculated exchange constants for $\left(\mathrm{V}_{0.96} \mathrm{Cr}_{0.04}\right)_{2} \mathrm{O}_{3}$ in the AFI phase with $U=3 \mathrm{eV}$ are shown in the fourth column of Table I. Overall, the in-plane interactions obtained from the spin-wave fit to the INS measurements are consistently accounted for by DFT while next-nearest-neighbor exchange interactions linking neighboring pseudohoneycomb lattice planes seem to be less well reproduced.

The simulated $\mathcal{S}(\mathbf{Q}, \omega)$ from these ab initio exchange parameters for $\left(\mathrm{V}_{0.96} \mathrm{Cr}_{0.04}\right)_{2} \mathrm{O}_{3}$ using LSWT as implemented in SPINW is shown in Fig. 3(a). Considering that this is a first-principles result, the similarity with measured INS in Fig. 3(c) is rather good. Limitations of DFT and LSWT for this low-spin $(S=1)$ quantum magnet may contribute to discrepancies between the experimental data and theory.

\section{Exchange interactions in the PI phase}

Having shown that DFT calculated exchange interactions in the AFI phase of $\left(\mathrm{V}_{0.96} \mathrm{Cr}_{0.04}\right)_{2} \mathrm{O}_{3}$ provide a good account of the measured spin-wave spectra, we use the same method to determine the exchange interactions in the PI phase, where the absence of coherent spin-wave excitations makes it harder to infer the exchange interactions from neutron scattering measurements.

DFT total energy calculations for the rhombohedral PI phase were performed using the high-temperature structure of Rozier et al. [60]. Table II shows the results for the first six exchange constants (identified by their bond distances) for pure $\mathrm{V}_{2} \mathrm{O}_{3}$ (column 4) and for $\mathrm{V}_{2} \mathrm{O}_{3}$ including $\mathrm{Cr}$ doping (column 5). The latter calculations were performed because, as mentioned earlier, we found this to be important

TABLE II. DFT calculated exchange constants (in meV) for the PI phase of pure $\mathrm{V}_{2} \mathrm{O}_{3}$ and $\left(\mathrm{V}_{0.96} \mathrm{Cr}_{0.04}\right)_{2} \mathrm{O}_{3}$. Column 3 shows the corresponding exchange constants for the lower-symmetry AFI phase.

\begin{tabular}{lcccc}
\hline \hline & & & DFT & DFT \\
\cline { 3 - 5 } Distance $(\AA)$ & $J_{j}$ & $\equiv J_{i}$ & Pure $\mathrm{V}_{2} \mathrm{O}_{3}$ & $\left(\mathrm{~V}_{0.96} \mathrm{Cr}_{0.04}\right)_{2} \mathrm{O}_{3}$ \\
\hline 2.71072 & $J_{1}$ & $J_{\alpha}$ & $2.7(5)$ & $-0.3(6)$ \\
2.87799 & $J_{2}$ & $J_{\beta 1}, J_{\beta 2}, J_{\gamma}$ & $9.3(2)$ & $8.5(3)$ \\
3.46255 & $J_{3}$ & $J_{\epsilon}, J_{\delta}$ & $2.1(2)$ & $0.6(3)$ \\
3.68774 & $J_{4}$ & $J_{\eta}, J_{\zeta 1}, J_{\zeta 2}$ & $0.7(1)$ & $0.0(2)$ \\
4.29734 & $J_{5}$ & $J_{\theta}$ & $-0.3(5)$ & $-1.2(7)$ \\
4.94240 & $J_{6}$ & $J_{l}, J_{\kappa}$ & $1.9(1)$ & $1.7(2)$ \\
\hline \hline
\end{tabular}



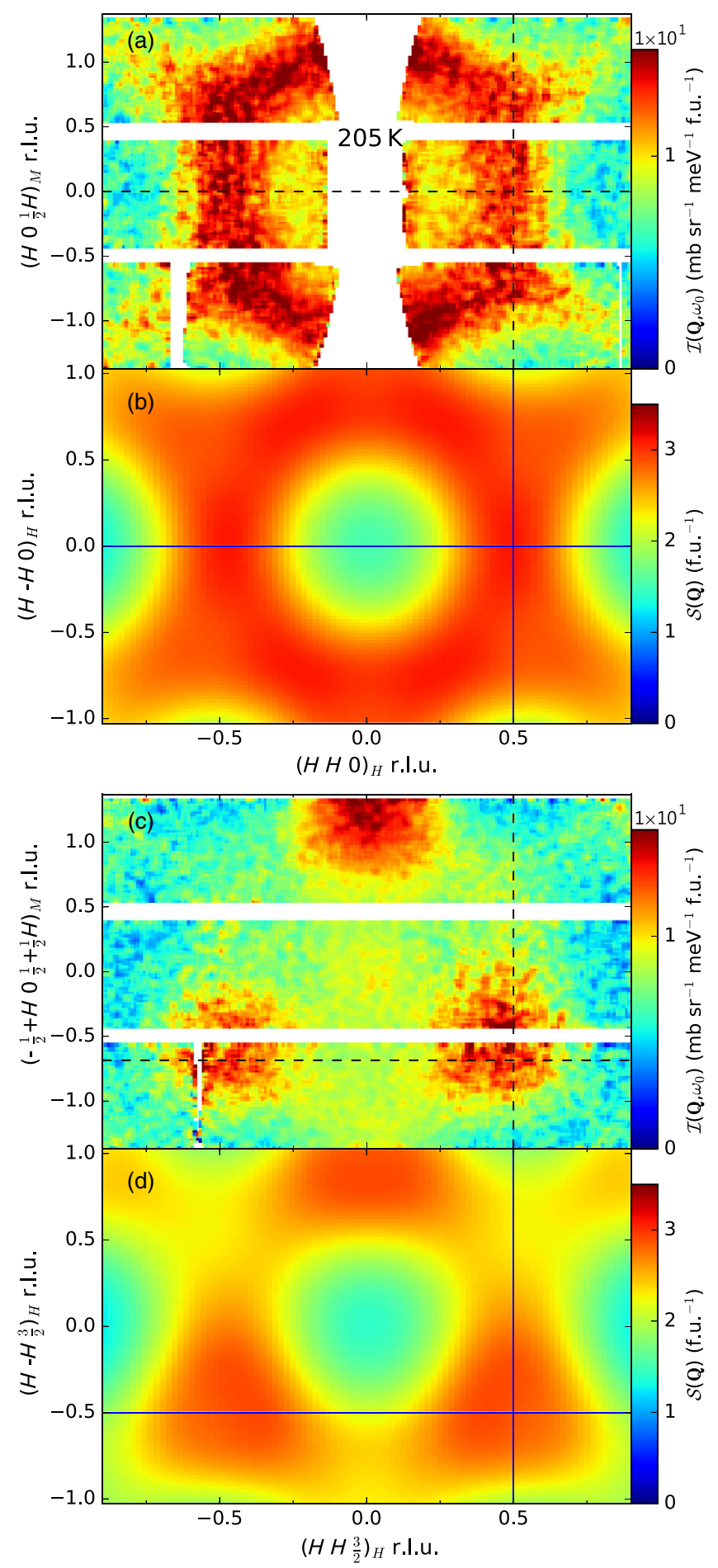

FIG. 4. (a),(c) Inelastic neutron scattering intensity from $\left(\mathrm{V}_{0.96} \mathrm{Cr}_{0.04}\right){ }_{2} \mathrm{O}_{3}$ at $205 \mathrm{~K}$ at $\hbar \omega_{0}=7.5 \mathrm{meV}$ (averaging range $=$ $[5,10] \mathrm{meV})$ for wave vector transfer within pseudohexagonal basal planes (as in Fig. 2) at offsets along the pseudohexagonal $c$ axis of $L_{H}=0$ (a),(b) and $L_{H}=1.5$ (c),(d). (b),(d) These experimental data are compared with the calculated dynamic spin-correlation function $\mathcal{S}(\mathbf{Q})$ obtained with the complete set of DFT calculated exchange constants of the PI phase for $\left(\mathrm{V}_{0.96} \mathrm{Cr}_{0.04}\right)_{2} \mathrm{O}_{3}$ given in Table II. Lines indicate positions of cuts shown in Fig. 6. particularly for the nearest-neighbor $J_{\alpha}$ interaction in the AFI phase. The corresponding exchange paths (bond vectors) are shown in Fig. 1(b). These exchange constants yield a Curie-Weiss temperature $\Theta_{\mathrm{CW}}$ of $-400 \mathrm{~K}$, which is consistent with the experimental value of $\Theta_{\mathrm{CW}}=-350 \mathrm{~K}$ for $\left(\mathrm{V}_{0.96} \mathrm{Cr}_{0.04}\right)_{2} \mathrm{O}_{3}\left(\Theta_{\mathrm{CW}}=-600 \mathrm{~K}\right.$ for pure $\left.\mathrm{V}_{2} \mathrm{O}_{3}\right)$ [67].

The fact that the magnetic correlation length is on the atomic scale (Fig. 4) even for $T<\frac{1}{2}\left|\Theta_{\mathrm{CW}}\right|$ indicates the PI phase is magnetically frustrated. Let us now examine whether or not the PI phase interactions inferred from DFT can account for the specific short-ranged nature of the spin correlations. The self-consistent Gaussian approximation (SCGA) was previously shown to be effective for determining the equal-time spin-correlation function $\mathcal{S}(\mathbf{Q})$ of geometrically frustrated magnets [69]. Based on a spherical spin model [70], the softened spin configurations are weighted by the Boltzmann factor $e^{-\beta E}$ with

$$
\beta E=\frac{1}{2} \sum_{i j}\left(\beta \sum_{n} J_{n} A_{i j}^{(n)}+\lambda \delta_{i j}\right) s_{i} s_{j} .
$$

Here $s_{i}$ denotes one component of the spin vector $\mathbf{S}_{i}$ and $A^{(n)}$ is the adjacency matrix between $n$th nearest neighbors. The Lagrangian multiplier $\lambda$ is determined self-consistently to ensure the average spin length $\left\langle\mathbf{S}_{i}^{2}\right\rangle=1$. The rhombohedral structure of the PI phase is broken into six hexagonal sublattices and the Fourier transform is taken to obtain a quadratic interaction between sublattices. In reciprocal space, the condition on $\lambda$ is expressed as the trace of the inverse interaction matrix $\left(\sum_{n} J_{n} A^{(n)}\right)$. The spin correlator as the two-body propagator is calculated at each desired $\mathbf{Q}$ point. We applied the SCGA method to determine spin correlations at $T=205 \mathrm{~K}$, where fluctuations of the classical spins are thermal. The calculated equal-time spin-correlation function $\mathcal{S}(\mathbf{Q})$ captures the dominant features of the experimental INS data (see the matching broad features shown in Figs. 4 and 6).

\section{ANALYSIS AND DISCUSSION}

\section{A. Frustrated interactions in the PI phase}

With the detailed set of exchange constants at hand (Table II), we are in a position to examine the nature of the frustrated PI phase. The two strongest exchange constants, $J_{2}$ and $J_{6}$ [see Figs. 1(b) and 5], form quasi-twodimensional honeycomb lattices with competing nearestneighbor $\left(J_{2}\right)$ and next-nearest-neighbor $\left(J_{6}\right)$ interactions. As can be observed in Fig. $6, J_{2}$ and $J_{6}$ are the two indispensable exchange constants for generating the observed $\mathcal{S}(\mathbf{Q})$ pattern, since the case of $J_{1}=J_{3}=J_{4}=$ $J_{5}=0$ only slightly modifies the $\mathcal{S}(\mathbf{Q})$ calculation.

While $J_{2}$ favors bipartite AFM order where each spin is antiparallel to its nearest neighbors, $J_{6}$ stabilizes a stripy phase [68]. For $\left(\mathrm{V}_{0.96} \mathrm{Cr}_{0.04}\right)_{2} \mathrm{O}_{3}$, the DFT calculations yield $J_{6} / J_{2} \sim 0.20$, which place the PI phase in the 


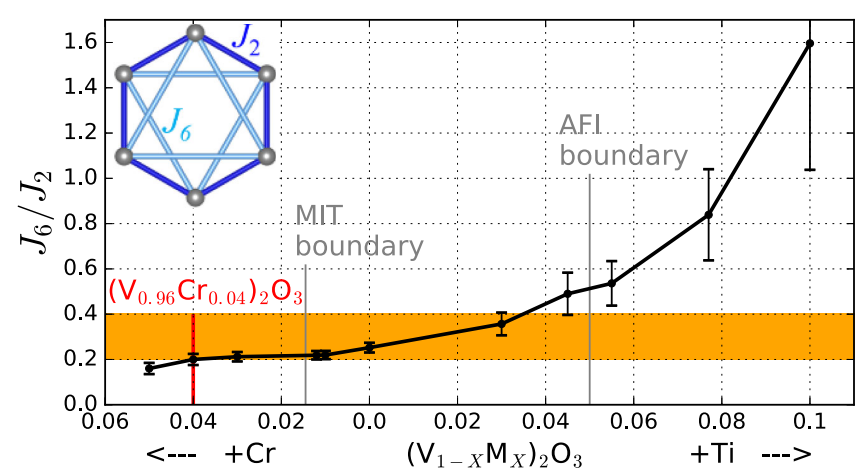

FIG. 5. The ratio of the in-plane honeycomb lattice nextnearest- and nearest-neighbor exchange interaction values, $J_{6} / J_{2}$, throughout the phase diagram [Fig. 1(a)] of $\mathrm{V}_{2} \mathrm{O}_{3}$ obtained from DFT calculations. The shaded orange region indicates the theoretically predicted range of frustrated interactions [68]. It is clear that changes in the crystal structure induced from $\mathrm{Cr}$ doping have only minor effects on $J_{6} / J_{2}$, as can also been seen in Table II.

valence bond crystal or spin liquid phase of the frustrated honeycomb model [68,71]. The correlation length for such a model may be sufficiently short that the only significant interlayer interactions (which are $J_{5}, J_{3}$, and $J_{1}$ in order of their strength) are rendered ineffective. Indeed, we note that the only out-of-plane exchange constant $J_{5}=-1.2(7)$, which is comparable in magnitude to $J_{6}$, does not substantially alter the pattern of magnetic excitations, and therefore does not appear to interfere with the frustration originating in the honeycomb basal plane. The importance of the next-nearest-neighbor interaction $J_{6}$ within the honeycomb layers of pure $\mathrm{V}_{2} \mathrm{O}_{3}$ was previously noted based on $\mathrm{GGA}+U$ calculations [23].

In order to estimate how widely this fundamental frustration mechanism is distributed over the PI phase, we again employed the virtual crystal approximation to perform DFT total energy calculations for different doping levels (with both $\mathrm{Cr}$ and $\mathrm{Ti}$ ). The resulting $J_{6} / J_{2}$ values versus $\mathrm{Cr}$ and $\mathrm{Ti}$ doping are shown in Fig. 5. These results indicate that pure $\mathrm{V}_{2} \mathrm{O}_{3}$ lies near the optimal ratio of $J_{6} / J_{2}$ for frustrated interactions, and that this frustrated configuration remains present up to doping levels of $\sim 4 \%$ with either $\mathrm{Cr}$ or Ti.

Examining the Q- $E$ slices for the PI phase in Fig. 3(d) reveals factorization of the $\mathbf{Q}$ and $E$ dependence of the dynamic spin-correlation function with a $40 \mathrm{meV}$ bandwidth in energy that resembles the Weiss temperature and greatly exceeds $k_{B} T$. Comparison of Figs. 3(c) and 3(d) shows this is not diffuse scattering of the AFI phase as it is weak at AFM Bragg points. Instead, such dynamic correlations resemble a number of strongly frustrated magnets that are described as quantum paramagnets, such as $\mathrm{ZnCr}_{2} \mathrm{O}_{4}[6]$ and $\mathrm{SrCr}_{9 p} \mathrm{Ga}_{12-9 p} \mathrm{O}_{19}$ [72].

The SCGA method breaks down at low temperatures when the interacting spin model develops magnetic order. A finite-size scaling calculation [using all the LDA $+U$
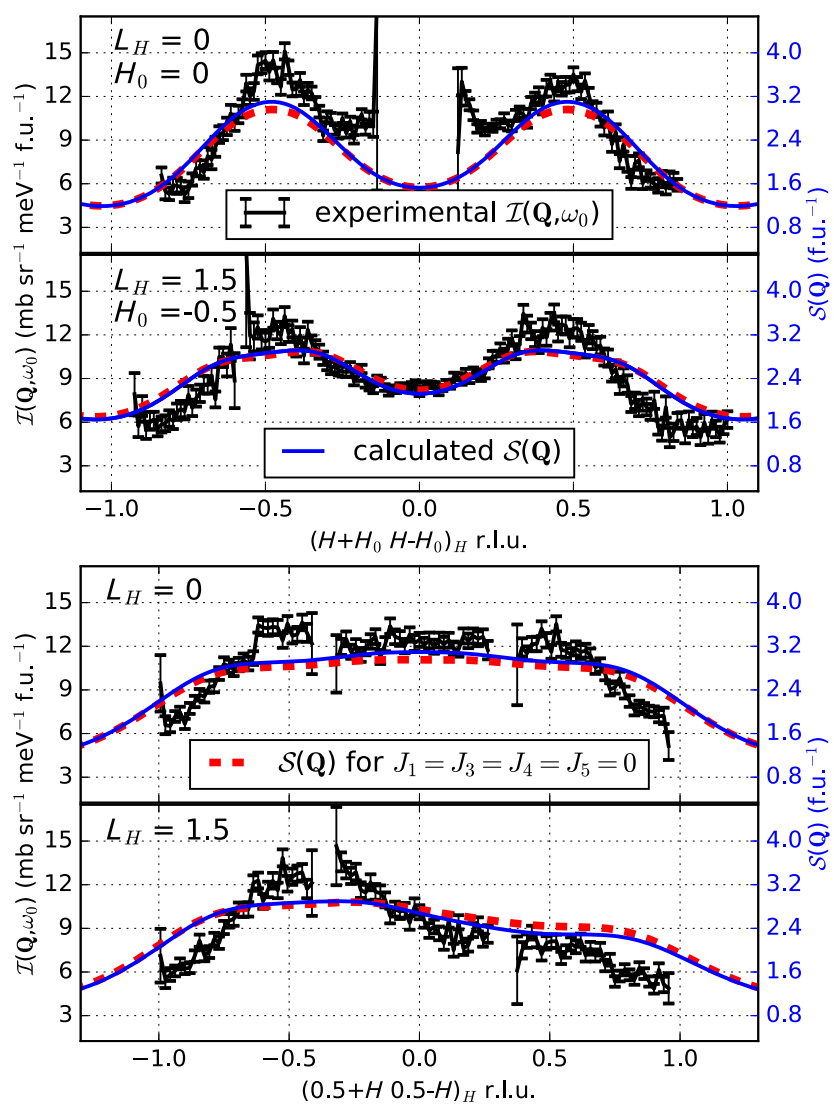

FIG. 6. Cuts through the experimental $\mathcal{I}\left(\mathbf{Q}, \omega_{0}\right)$ (black error bars) and the calculated $\mathcal{S}(\mathbf{Q})$ (blue lines) for $\left(\mathrm{V}_{0.96} \mathrm{Cr}_{0.04}\right)_{2} \mathrm{O}_{3}$. Top (bottom) panels show horizontal (vertical) cuts taken along the corresponding lines indicated in Fig. 4. To show the importance of the dominant exchange constants $J_{2}=8.5(3)$ and $J_{6}=1.7(2)$, the red line shows the calculated $\mathcal{S}(\mathbf{Q})$ when $J_{1}=J_{3}=J_{4}=J_{5}=0$.

calculated exchange constants for $\left(\mathrm{V}_{0.96} \mathrm{Cr}_{0.04}\right)_{2} \mathrm{O}_{3}$ in Table II] estimates the ordering temperature to be $\sim 10 \mathrm{~K}$. Comparison to the Curie-Weiss temperature of $\sim 400 \mathrm{~K}$ yields a frustration index of $f=40$, indicating a high degree of magnetic frustration in the PI phase. The $T \approx 10 \mathrm{~K}$ phase transition anticipated for the PI spin Hamiltonian is, however, preempted in $\left(\mathrm{V}_{0.96} \mathrm{Cr}_{0.04}\right)_{2} \mathrm{O}_{3}$ at $T_{N}=185 \mathrm{~K}$ by the first-order magnetostructural transition that relieves magnetic frustration and gains magnetic exchange energy at the expense of lattice strain energy. DFT calculations and neutron scattering measurements indicate that the magnetic exchange interactions are significantly modified at this transition (compare Tables I and II). In particular, the three identical nearest-neighbor interactions within the PI honeycomb lattices [DFT, $\left.J_{2}=8.5(3) \mathrm{meV}\right]$ split into three distinct interactions in the AFI phase [DFT[neutron], $J_{\beta_{1}}=25(2)[27.7(2)] \mathrm{meV}$, $J_{\beta_{2}}=9(2)[7.7(2)] \mathrm{meV}$, and $\left.J_{\gamma}=3(2)[0.0(2)] \mathrm{meV}\right]$. This relieves frustration and gives way to the stripy ordered phase favored by the next-nearest-neighbor interaction [DFT, $J_{6}=1.7(2) \mathrm{meV}$ ]. 


\section{B. Unfrustrated interactions in the AFI phase}

Referring to the experimental exchange constants in the fifth column of Table I, we note that they are consistently unfrustrated: Spins that interact antiferromagnetically (ferromagnetically) are also antiferromagnetically (ferromagnetically) correlated in the ordered state (see column 6 of Table I). Thus, while exchange interactions in the PI phase realize a frustrated honeycomb antiferromagnet with competing nearest- $\left(J_{2}\right)$ and next-nearest-neighbor $\left(J_{6}\right)$ antiferromagnetic interactions, the modified nearest-neighbor interactions in the AFI phase are all simultaneously satisfied in the long-range-ordered magnetic state (i.e., the signs of the fifth and sixth columns of Table I are consistently opposite).

The raw experimental evidence for unfrustrated interactions is the large bandwidth of magnetic excitations in the AFI phase $(80 \mathrm{meV})$. Because the bandwidth exceeds $k_{B} T_{N}$ by a factor of 4 , the collapse of the ordered state upon heating occurs before significant thermal population of spin waves. Correspondingly, there is no buildup of magnetic correlations upon cooling the frustrated PI phase towards the $T_{N}=185 \mathrm{~K}$ phase transition. Comparison of Figs. 3(c) and 3(d) shows a clear upward shift of magnetic spectral weight, which indicates lowering of the magnetic exchange energy [6] as frustration is relieved at the first-order PI-toAFI transition.

The nearest-neighbor interplane interaction between face-sharing vanadium atoms, $J_{\alpha}=-6.0(2) \mathrm{meV}$, is the main FM interaction that favors FM sheets in the AFI phase. Our results confirm that $J_{\beta}$ and $J_{\zeta}$ are stronger than $J_{\alpha}$, and therefore suggest that superexchange via $\mathrm{V}-\mathrm{O}-\mathrm{V}$ paths contributes to the realization of a strong ferromagnetic alignment for the vertical $\mathrm{V}$ pairs. The values of $J_{\alpha}=-4.1 \mathrm{meV}$ and $J_{\beta 1}=18.4 \mathrm{meV}$ determined by the LDA $+U$ study of Ref. [40] are consistent with these values. Further, the suggestion of Ref. [40] that the nextnear-neighbor exchange interactions are significant agrees with our result of $J_{\zeta}=7.1(2) \mathrm{meV}$.

It is interesting to compare the experimental and $a b$ initio exchange interactions for $\left(\mathrm{V}_{0.96} \mathrm{Cr}_{0.04}\right)_{2} \mathrm{O}_{3}$ with those of pure $\mathrm{Cr}_{2} \mathrm{O}_{3}$. Samuelsen et al. [73] showed that only the first two exchange interactions are significant in $\mathrm{Cr}_{2} \mathrm{O}_{3}$, with the nearest-neighbor interaction dominant at $7.5 \mathrm{meV}$. Thus, the range of interactions in $\left(\mathrm{V}_{0.96} \mathrm{Cr}_{0.04}\right)_{2} \mathrm{O}_{3}$ is considerably longer than in $\mathrm{Cr}_{2} \mathrm{O}_{3}$, which is broadly consistent with greater $3 d$-electron delocalization near the Mott transition of $\mathrm{V}_{2} \mathrm{O}_{3}$.

In addition to indicating a transition from a frustrated to an unfrustrated magnet, the doubling of the magnetic bandwidth from 40 to $80 \mathrm{meV}$ during the PI-AFI transition serves as another sign of its first-order nature [3]. Tables I and II show that the magnetic exchange interactions are tightly coupled with the crystal structure and lattice parameters. Therefore, spin-phonon coupling is expected in $\mathrm{V}_{2} \mathrm{O}_{3}$ and is indeed evidenced in Brillouin scattering studies [74]. Comparison of Figs. 3(c) and 3(d) shows phonon excitations near $70-80 \mathrm{meV}$, which appear to overlap with optical magnon branches. The intensity enhancement of the magnon modes in this region compared with the LSWT structure factor shown in Fig. 3(b) could be a result of magnon-phonon coupling. It is also likely that the overall dispersion of the phonon normal modes is significantly affected by both spin-phonon and magnon-phonon interactions. To quantify these effects as well as the relationship between the magnetic interactions and the PI-AFI phase transition, it will be interesting future work to calculate quantitative values for the magnetoelastic coupling strengths. Magnetoelastic effects near room temperature present great opportunities for applications. Strain in $\mathrm{V}_{2} \mathrm{O}_{3}$ thin films has, for example, been shown to influence the metal-insulator transition [75,76], and the coercive field of nickel thin films deposited on $\mathrm{V}_{2} \mathrm{O}_{3}$ is almost doubled at the MIT $[77,78]$.

\section{CONCLUSION}

In summary, magnetic exchange interactions in the AFI phase of $\left(\mathrm{V}_{0.96} \mathrm{Cr}_{0.04}\right)_{2} \mathrm{O}_{3}$ were determined with INS measurements of both acoustic and optic spin waves throughout the monoclinic Brillouin zone. Ordered by magnitude, the leading interactions are $J_{\beta}$ and $J_{\zeta}$, which are both antiferromagnetic, and the nearest-neighbor interlayer interaction $J_{\alpha}$, which is ferromagnetic [Fig. 1(c)]. These and indeed all interactions in the AFI phase are satisfied within the observed low- $T$ magnetic structure. In the PI phase, diffuse inelastic magnetic neutron scattering and DFT calculations combined with a Gaussian approximation to the spin-correlation function show this is a quasi-two-dimensional cooperative paramagnet with frustrated nearest- $\left(J_{2}\right)$ and next-nearestneighbor $\left(J_{6}\right)$ interactions within the puckered honeycomb layers that form the corundum structure [Fig. 1(b)]. We infer that the strong suppression of magnetic order that results from this frustration is key to preventing the AFI phase from engulfing the Mott-like paramagnetic metal-to-insulator phase boundary in $\mathrm{V}_{2} \mathrm{O}_{3}$.

The insulating states of compounds with an experimentally accessible Mott-like phase boundary including $\mathrm{Ni}\left(\mathrm{S}_{1-x} \mathrm{Se}_{x}\right)_{2}$ [79], $\mathrm{GaTa}_{4} \mathrm{Se}_{8}$ [80,81], $\mathrm{Na}_{4} \mathrm{Ir}_{3} \mathrm{O}_{8}$ $[82,83]$, and the quasi-two-dimensional organic systems $\kappa-(\mathrm{ET})_{2} \mathrm{Cu}\left[\mathrm{N}(\mathrm{CN})_{2}\right] \mathrm{Cl}, \kappa-(\mathrm{ET})_{2} \mathrm{Cu}_{2}(\mathrm{CN})_{3} \quad$ [84] and $\mathrm{EtMe}_{3} \mathrm{Sb}\left[\mathrm{Pd}(\mathrm{dmit})_{2}\right]_{2}$ [11] have all been found to be magnetically frustrated. Conversely, the spin-liquid-like properties of the latter two compounds have been associated with the increased range and nature of spin-spin interactions near the Mott transition. The present work on $\left(\mathrm{V}_{1-x} \mathrm{Cr}_{x}\right)_{2} \mathrm{O}_{3}$ reinforces these interesting links between frustrated magnetism and the Mott transition, and signifies that the ongoing searches for a Mott insulator with a paramagnetic MIT are tied to the search for spin liquids $[85,86]$.

\section{ACKNOWLEDGMENTS}

The authors would like to acknowledge helpful discussions with Sandor Toth, Arnab Banerjee, Michael Lawler, 
and Oleg Tchernyshyov. R. V. thanks Frank Lechermann for useful discussions. This project was supported by UT-Battelle LDRD No. 3211-2440. Work at IQM was supported by the U.S. Department of Energy, Office of Basic Energy Sciences, Division of Materials Sciences and Engineering through Grant No. DE-SC0019331. This research used resources at the Spallation Neutron Source, a DOE Office of Science User Facility operated by the Oak Ridge National Laboratory (ORNL). C. L. B. was supported through the Gordon and Betty Moore foundation under the EPIQS program GBMF4532. This research used resources of the National Energy Research Scientific Computing Center, a DOE Office of Science User Facility supported by the Office of Science of the U.S. Department of Energy under Contract No. DE-AC0205CH11231. Work of J.H. and O.D. at ORNL was supported by the U.S. Department of Energy, Office of Basic Energy Sciences, Division of Materials Sciences and Engineering through Grant No. DE-SC0016166. The work at BIT was supported by the National Science Foundation of China Grant No. 11572040. W. B. was supported by National Natural Science Foundation of China (Grant No. 11227906). The work in Frankfurt was supported by the Deutsche Forschungsgemeinschaft under Grant No. SFB/TRR 49.

\section{APPENDIX A: DATA PROCESSING METHODS}

\section{Incoherent background subtraction}

With the new capabilities of the MANTID data analysis software [58], it is possible to subtract a $\phi$-independent ( $\phi$ being the azimuthal rotation angle of $\mathbf{k}_{f}$ around $\mathbf{k}_{i}$ ) background signal from the 4D $\mathcal{S}(\mathbf{Q}, \omega)$ data. This is done by taking a judiciously sampled powder average of the $\mathcal{S}(\mathbf{Q}, \omega)$ including only incoherent $\phi$-independent components and excluding coherent $\phi$-dependent components (see Fig. 7). Once this special powder average is obtained, it can readily be subtracted from the $\mathcal{S}(\mathbf{Q}, \omega)$ data as a background.

\section{Phonon density of states calculations}

Normalization of the scattering data was achieved through the incoherent phonon scattering cross section. The neutron weighted phonon DOS $g^{(n)}(\omega)$ was theoretically calculated with VASP + PHONOPY for the AFI phase of $\mathrm{V}_{2} \mathrm{O}_{3}$. This is shown as a solid green line in Fig. 8 in units of $\mathrm{meV}^{-1}$ f.u. $^{-1}$ (5 atoms per formula unit).

The experimental phonon DOS was obtained by first taking a constant $Q$ cut through the effective powder average (generated by averaging over all collected sample rotation angles) of the $T=5 \mathrm{~K}$ INS spectra. In this case the cut is taken by integrating in a rectangular area at high momentum transfer centered around $|Q|=5.3 \AA^{-1}$, as shown by the dashed red lines in Fig. 7(a). The result of this integration is plotted as the dashed red line (not to scale) in Fig. 8. This cut

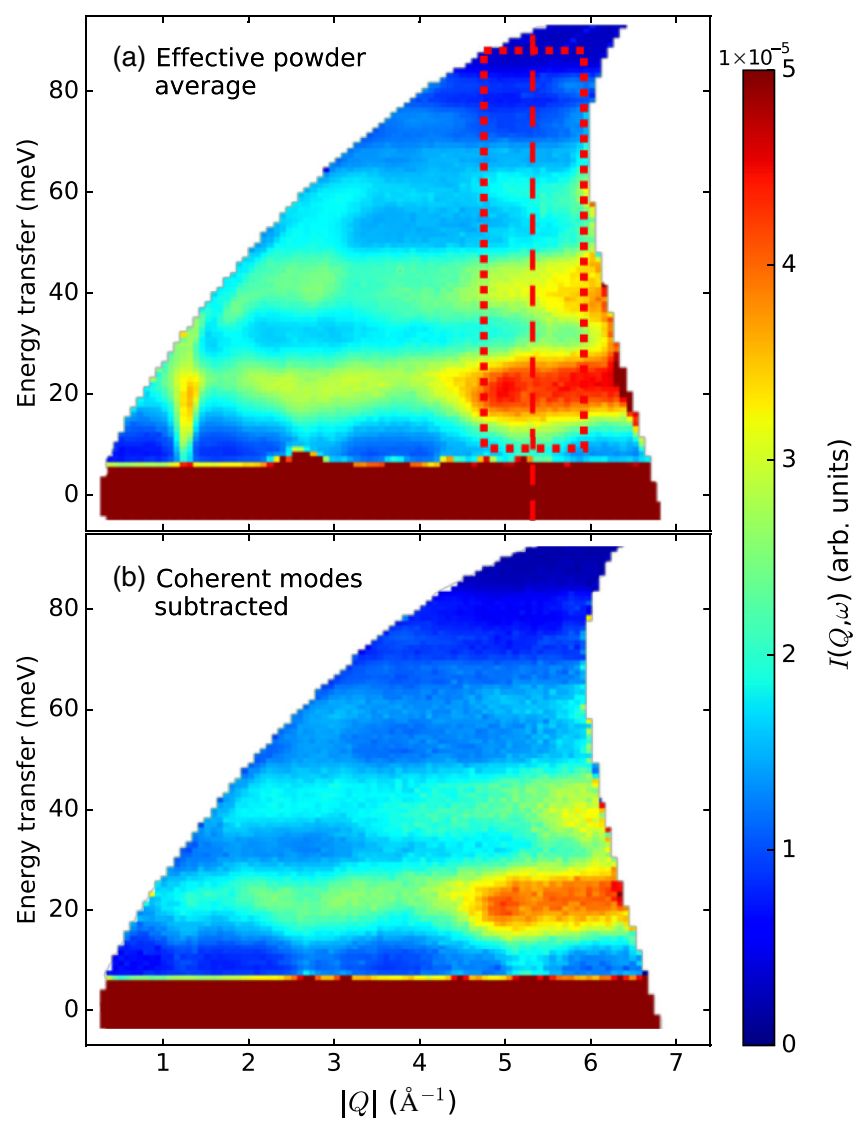

FIG. 7. (a) An effective powder average (taken over all collected sample rotation angles) for the single-crystal $\left(\mathrm{V}_{0.96} \mathrm{Cr}_{0.04}\right)_{2} \mathrm{O}_{3} \mathrm{~T}=5 \mathrm{~K}$ data. The dashed red lines indicate the range of wave vector transfer used to generate the phonon DOS shown in Fig. 8. (b) Powder average of the same data excluding scattering that depends on the azimuthal angle $\phi$. Such filtered data were utilized as background.

from the experimental data can then be converted into the neutron weighted generalized phonon DOS $g^{(n)}(\omega)$ (the blue line in Fig. 8) via the following formula:

$$
g^{(n)}(\omega)=\frac{\mathcal{A} I(Q, \omega)}{\frac{\sigma_{\mathrm{V}_{2} \mathrm{O}_{3}}}{4 \pi}\left(\frac{\hbar Q)^{2}}{2 M} / \hbar \omega\right)[n(\omega, T)+1]},
$$

where $I(Q, \omega)$ is the proton charge normalized detector counts binned in $Q=|\mathbf{Q}|$ and $\omega, n(\omega, T)=\left[e^{\beta \omega}-1\right]^{-1}$, $\sigma_{\mathrm{V}_{2} \mathrm{O}_{3}}=22.88 \mathrm{~b} / \mathrm{f}$.u. is the total scattering cross section (coherent plus incoherent) per formula unit for the sample, and $M$ is the mass per formula unit. $\mathcal{A}$ is the normalization constant, which was adjusted to achieve the best overlap between the experimental and theoretical $g^{(n)}(\omega)$ traces in Fig. 8. This procedure yields $\mathcal{A}=$ $200(50) \mathrm{bC} \mathrm{meV}^{-1}$ counts $^{-1}$. Using this normalization factor the conversion of raw scattering intensities to a normalized cross section is as follows: $\mathcal{I}(\mathbf{Q}, \omega)=$ $\mathcal{A} I(\mathbf{Q}, \omega)$. 


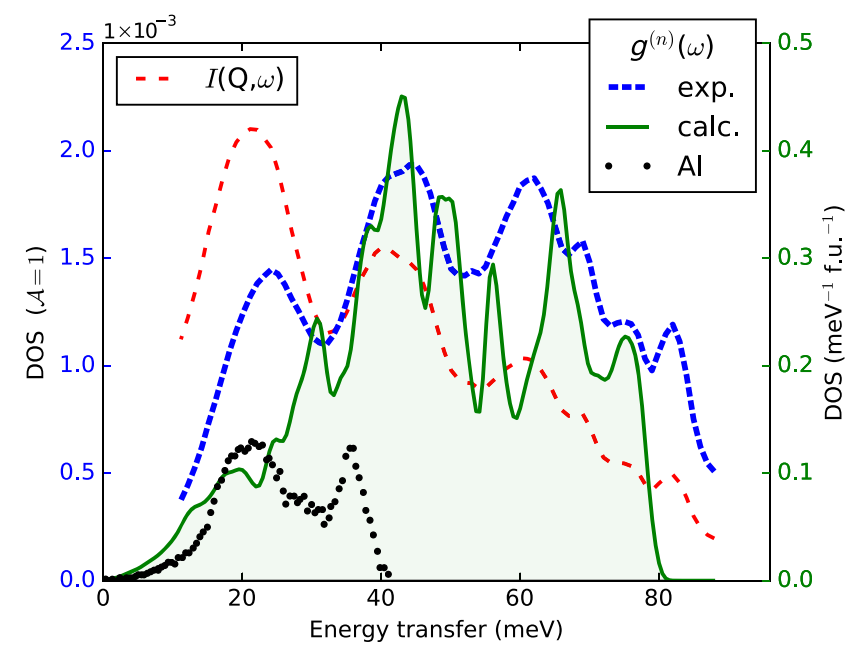

FIG. 8. Phonon DOS $g^{(n)}(\omega)$ for $\left(\mathrm{V}_{0.96} \mathrm{Cr}_{0.04}\right)_{2} \mathrm{O}_{3}$ extracted from the effective powder average of the INS data [see Fig. 7(a)]. This is compared with theoretical phonon DOS calculated for $\mathrm{V}_{2} \mathrm{O}_{3}$ in the low-temperature AFI phase. Also shown is the phonon DOS for aluminum from Ref. [87]. A contribution to the experimental data from aluminum is inevitable due to the aluminum sample mount and cryostat windows.

Some of the discrepancy between the experimental and theoretical $g^{(n)}(\omega)$ may arise from contributions to scattering intensity from the aluminum can sample holder. The previously measured phonon DOS for aluminum [87] shows its strongest peak is near $20 \mathrm{meV}$ energy transfer. This is consistent with the extra intensity seen near $20 \mathrm{meV}$ in the experimental $g^{(n)}(\omega)$ when comparing with the theoretical $g^{(n)}(\omega)$ for $\mathrm{V}_{2} \mathrm{O}_{3}$. We estimate that this normalization procedure is accurate to within $25 \%$.

\section{APPENDIX B: EXTENDED DATA MODELING}

\section{DFT calculated exchange constants}

The initial DFT calculations of exchange interactions were performed for pure monoclinic $\mathrm{V}_{2} \mathrm{O}_{3}$. These yielded results with smaller statistical errors than when including the effects of chromium doping. As mentioned in the main text, these calculations were performed with the full potential local orbital (FPLO) basis set [61] and the GGA functional [62]. Total energies for large sets of different spin configurations were calculated with GGA $+U$ using the atomic limit double-counting correction [88]. We fix $J_{H}=0.68 \mathrm{eV}$ [89] and vary $U$. For the LSWT modeling, we employ only the $U=3 \mathrm{eV}$ values.

The calculation was carried out to obtain the first 12 exchange constants of monoclinic pure $\mathrm{V}_{2} \mathrm{O}_{3}$ by combining the total energies of two supercells: (1) a $2 \times 2 \times 2$ supercell with $P \overline{1}$ symmetry, which leaves $8 \mathrm{~V}^{3+}$ ions inequivalent, and (2) a $1 \times \sqrt{2} \times \sqrt{2}$ with $P \overline{1}$ symmetry, also containing 8 inequivalent $\mathrm{V}^{3+}$ ions. While neither of the two structures
TABLE III. Exchange constants for monoclinic pure $\mathrm{V}_{2} \mathrm{O}_{3}$ (in $\mathrm{meV}$ ) calculated with DFT using two unit cells as explained in the text below. The resultant INS cross sections from the spin waves with these exchange constants are shown in Fig. 9(a). Also, shown in Fig. 9(b) is the LSWT result using the set of DFT calculated exchange parameters from Table I for $\left(\mathrm{V}_{0.96} \mathrm{Cr}_{0.04}\right)_{2} \mathrm{O}_{3}$ with adjustments made (as described in the text) within statistical error tolerances, except for $J_{\zeta 2}$ (shown in bold). $J_{\zeta 2}$ must be increased to $6.5 \mathrm{meV}$, which is outside of the statistical error bar interval, to achieve the level of agreement with INS reflected by Fig. 9(b).

\begin{tabular}{lccc}
\hline \hline$J_{i}$ & Distance $(\AA)$ & Pure $\mathrm{V}_{2} \mathrm{O}_{3}$ & Fig. 9(b) \\
\hline$J_{\alpha}$ & 2.75904 & $2.8(3)$ & -2.7 \\
$J_{\beta 1}$ & 2.83083 & $28.7(3)$ & 27.5 \\
$J_{\beta 2}$ & 2.91789 & $12.4(3)$ & 7.7 \\
$J_{\gamma}$ & 2.98538 & $-2.3(3)$ & 2.8 \\
$J_{\epsilon}$ & 3.43336 & $-3.9(5)$ & -6.1 \\
$J_{\delta}$ & 3.45420 & $3.5(3)$ & 1.9 \\
$J_{\eta}$ & 3.63334 & $0.6(3)$ & -1.0 \\
$J_{\zeta 1}$ & 3.70177 & $2.5(2)$ & 2.5 \\
$J_{\zeta 2}$ & 3.76876 & $-0.3(2)$ & $\mathbf{6 . 5}$ \\
$J_{\theta}$ & 4.22293 & $0.8(3)$ & -1.6 \\
$J_{l}$ & 4.97765 & $0.4(2)$ & 2.0 \\
$J_{\kappa}$ & 5.00240 & $1.7(2)$ & 0.0 \\
\hline \hline
\end{tabular}

allow for resolution of all 12 exchange constants, the combined equations have enough information.

The exchange constants resulting from this calculation at $U=3 \mathrm{eV}$ are listed in the third column of Table III. The equations determined for the spin configurations analyzed indicate some correlations between the inferred exchange constants, in particular between $J_{\alpha}$ and $J_{\epsilon}$. Statistical errors are on the order of $0.5 \mathrm{meV}$, providing a reasonable degree of confidence for all except the smallest exchange constants, $J_{\zeta 2}$ and $J_{l}$. The resultant neutron scattering intensity obtained from these exchange constants for pure $\mathrm{V}_{2} \mathrm{O}_{3}$ through LSWT as implemented in SPINW [59] is plotted in Fig. 9(a).

We now return to the DFT calculated exchange constants for $\left(\mathrm{V}_{0.96} \mathrm{Cr}_{0.04}\right)_{2} \mathrm{O}_{3}$ listed in Table I. As the statistical error in these is around $2 \mathrm{meV}$, liberty may be taken to make adjustments within these error ranges to bring the calculated spin-wave scattering into agreement with observations. Such optimally adjusted $J$ values are shown in column 4 of Table III. The resultant neutron intensity is plotted in Fig. 9(b). The only adjustment that must stray beyond the tolerances of the statistical errors from Table I in order to achieve consistency with experiment is the value of $J_{\zeta 2}$ (set as $6.5 \mathrm{meV}$, shown in bold). The DFT methods may have trouble accounting for this particular interaction due to accuracy limitations of the structural model for such long-range superexchange interactions. Nevertheless, this alternate set of exchange parameters yields a pattern of scattering that is consistent with the neutron data, which 


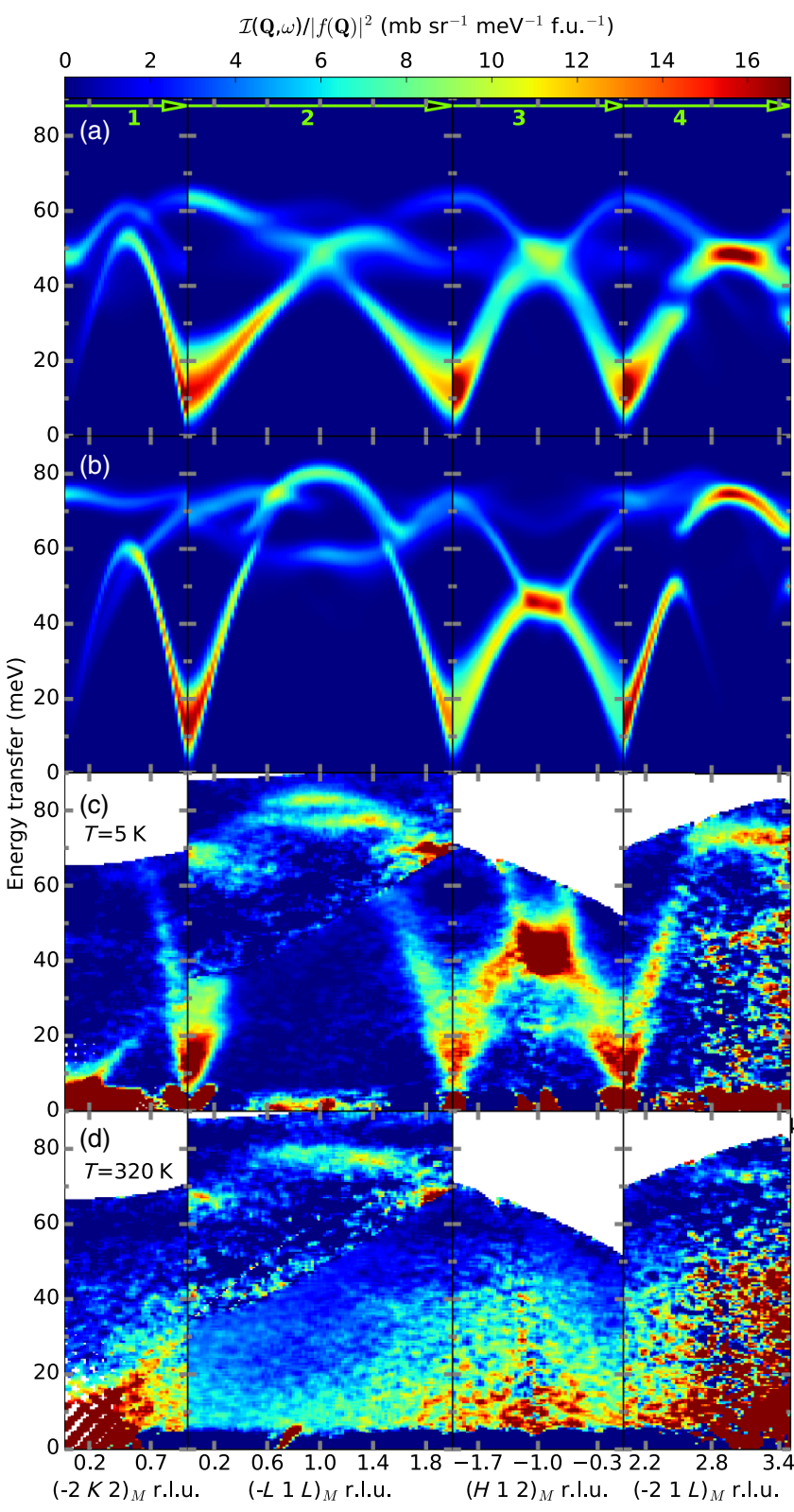

FIG. 9. (a),(b) Neutron scattering intensity associated with spin waves along four high-symmetry directions marked in Fig. 2 calculated with SPINW [59] for exchange constants shown in Table III. (c),(d) INS cross section measured at 5 and $320 \mathrm{~K}$ $\left(E_{i}=100 \mathrm{meV}\right)$. Data from multiple Brillouin zones were assembled to cover the largest possible range of energy transfer. As before, the data were divided by the squared magnetic form factor for presentation and the incoherent background was subtracted from the data (Appendix A 1).

indicates considerable correlated uncertainty in deriving exchange constants from scattering data. Comparison of column 5 in Table I and column 4 of Table III indicates the experimental error bar in the determination of these exchange constants from neutron scattering data. We note that in the original fitting we set $J_{\theta}=J_{l}=J_{\kappa}=0$ to reduce the number of fitting parameters. Clearly, with the use of 12 exchange constants to describe the neutron data (as with the $a b$ initio DFT calculations) instead of 9 for our standard direct fitting, we may expect increased correlated uncertainties in the extracted exchange constants. Nonetheless, the overall qualitative trends and magnitudes of the two presented sets of exchange constants which fit the data are similar, thus preserving the validity of the associated discussion in the main text.

\section{Resolution effects}

Looking at Figs. 3(a)-3(c) as well as Figs. 9(a)-9(c), more broadening is noticeable in the experimental data [Figs. 3(c) and 9(c)] of the acoustic magnon branches than is predicted by the simulations [Figs. 3(a), 3(b), 9(a), and 9(b)] with LSWT via SPINW. This discrepancy is present despite using consistent averaging ranges to produce each slice and the nominal energy resolution of SEQUOIA to approximate resolution effects within SPINW. To determine whether this reflects the physics of $\mathrm{V}_{2} \mathrm{O}_{3}$ or more intricate $\mathbf{Q}$-resolution effects, we performed Monte Carlo ray-tracing simulations of the experiment using MCVINE [90].

The simulation follows the routine MCVINE simulation procedure [90] that involves four steps. First, the neutron beam $\sim 12 \mathrm{~cm}$ upstream of the sample position was simulated. In the second step, the simulated beam was scattered by a virtual sample, which is a plate of $4.6 \times$ $4.6 \times 0.57 \mathrm{~cm}^{3}$ and has a scattering kernel of a dispersion surface (see the Supplemental Material of Ref. [90]) specified by an analytical dispersion function. The virtual sample is aligned as in the experiment and the goniometer angle was swept from $-90^{\circ}$ to $90^{\circ}$ in $2^{\circ}$ steps. In the third step, the interception of simulated scattered neutrons by the SEQUOIA [56] detector system was simulated and a series of NeXus files [91] were generated. In the last step, the NeXus files were reduced and slices were taken as for the real neutron scattering data.

The results are shown in Fig. 10. By examining constant energy cuts as shown in Fig. 11, one sees that the

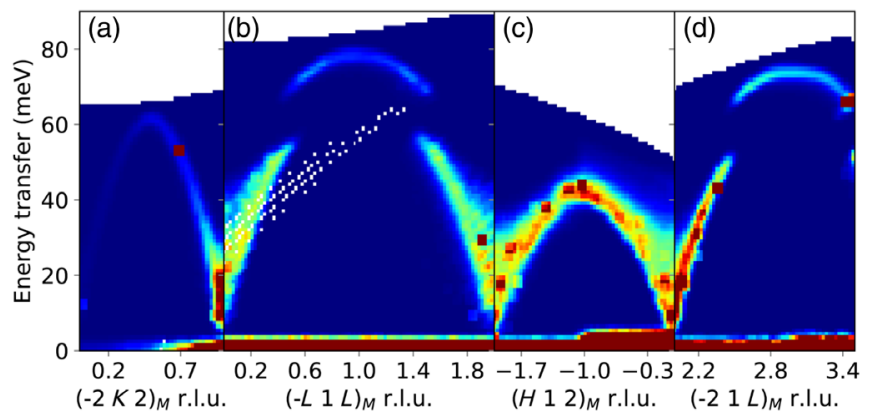

FIG. 10. MCVINE simulation of the same $\mathcal{I}(\mathbf{Q}, \omega)$ slices shown in Figs. 3(c) and 9(c). This simulation takes into account all instrumental effects and shows similar linewidth broadening as in the experimental scattering data. 

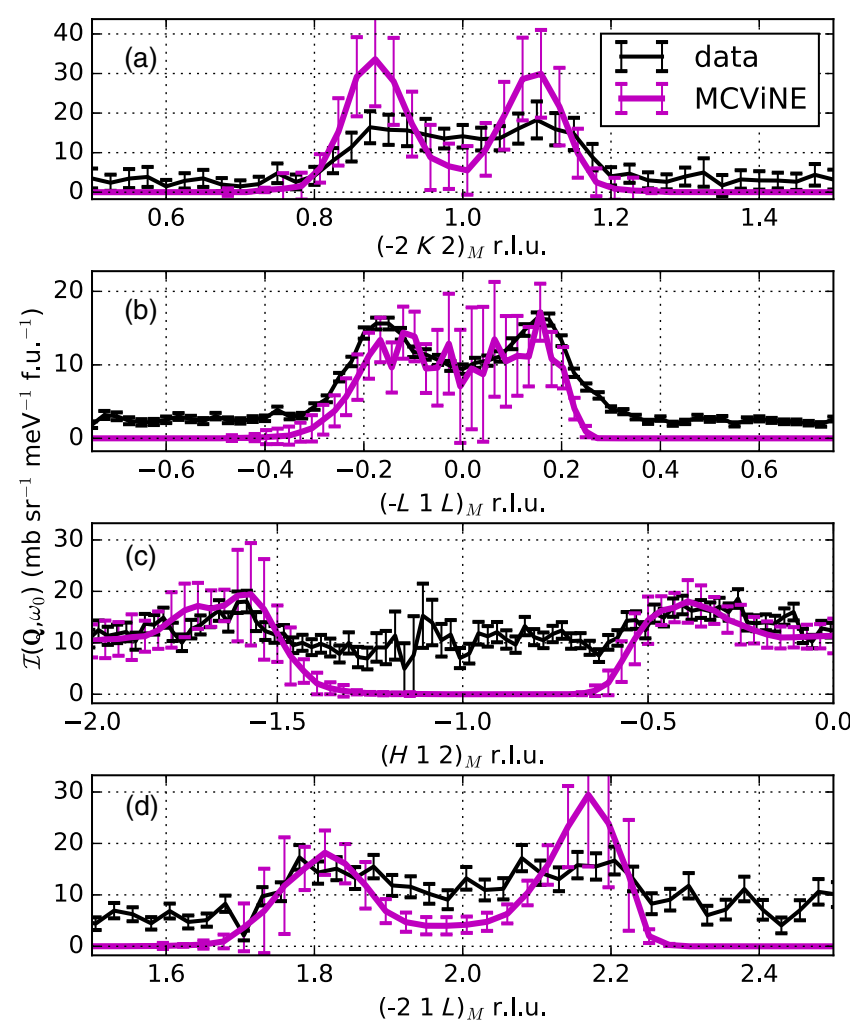

FIG. 11. Constant energy cuts at $\hbar \omega_{0}=30 \mathrm{meV}$ (averaging range $=[29,31] \mathrm{meV}$ ) through the experimental data for $\left(\mathrm{V}_{0.96} \mathrm{Cr}_{0.04}\right)_{2} \mathrm{O}_{3}$ at $5 \mathrm{~K}$ [Fig. 3(c)] and the corresponding MCVINE simulation (Fig. 10).

broadening of the acoustic spin waves in the actual neutron data is, for the most part, reproduced by this simulation of realistic instrumental effects. There is, however, a tendency towards sharper peaks in the simulation [especially in Figs. 11(a) and 11(d)]. This indicates some spin-wave damping, which is not unexpected for a quantum magnet with quenched disorder (due to chromium doping) near the MIT. But overall, the coherent magnon approximation provides a reasonable description of the full dataset.

[1] I. Leonov, V. I. Anisimov, and D. Vollhardt, Metal-Insulator Transition and Lattice Instability of Paramagnetic $\mathrm{V}_{2} \mathrm{O}_{3}$, Phys. Rev. B 91, 195115 (2015).

[2] A. I. Frenkel, D. M. Pease, J. I. Budnick, P. Metcalf, E. A. Stern, P. Shanthakumar, and T. Huang, Strain-Induced Bond Buckling and Its Role in Insulating Properties of Cr-Doped $\mathrm{V}_{2} \mathrm{O}_{3}$, Phys. Rev. Lett. 97, 195502 (2006).

[3] B. A. Frandsen, L. Liu, S. C. Cheung, Z. Guguchia, R. Khasanov, E. Morenzoni, T. J. S. Munsie, A. M. Hallas, M. N. Wilson, Y. Cai et al., Volume-Wise Destruction of the Antiferromagnetic Mott Insulating State through Quantum Tuning, Nat. Commun. 7, 12519 (2016).

[4] F. Lechermann, N. Bernstein, I. I. Mazin, and R. Valentí, Uncovering the Mechanism of the Impurity-Selective Mott
Transition in Paramagnetic $\mathrm{V}_{2} \mathrm{O}_{3}$, Phys. Rev. Lett. 121, 106401 (2018).

[5] O. Tchernyshyov, R. Moessner, and S. L. Sondhi, Order by Distortion and String Modes in Pyrochlore Antiferromagnets, Phys. Rev. Lett. 88, 067203 (2002).

[6] S.-H. Lee, C. Broholm, T. H. Kim, W. Ratcliff, and S-W. Cheong, Local Spin Resonance and Spin-Peierls-like Phase Transition in a Geometrically Frustrated Antiferromagnet, Phys. Rev. Lett. 84, 3718 (2000).

[7] S.-H. Lee, D. Louca, H. Ueda, S. Park, T. J. Sato, M. Isobe, Y. Ueda, S. Rosenkranz, P. Zschack, J. Íñiguez, Y. Qiu, and R. Osborn, Orbital and Spin Chains in $\mathrm{ZnV}_{2} \mathrm{O}_{4}$, Phys. Rev. Lett. 93, 156407 (2004).

[8] S.-H. Lee, H. Takagi, D. Louca, M. Matsuda, S. Ji, H. Ueda, Y. Ueda, T. Katsufuji, J.-H. Chung, S. Park, S.-W. Cheong, and C. Broholm, Frustrated Magnetism and Cooperative Phase Transitions in Spinels, J. Phys. Soc. Jpn. 79, 011004 (2010).

[9] Z. A. Kelly, M. J. Gallagher, and T. M. McQueen, Electron Doping a Kagome Spin Liquid, Phys. Rev. X 6, 041007 (2016).

[10] I. I. Mazin, H. O. Jeschke, F. Lechermann, H. Lee, M. Fink, R. Thomale, and R. Valentí, Theoretical Prediction of a Strongly Correlated Dirac Metal, Nat. Commun. 5, 4261 (2014).

[11] T. Furukawa, K. Miyagawa, H. Taniguchi, R. Kato, and K. Kanoda, Quantum Criticality of Mott Transition in Organic Materials, Nat. Phys. 11, 221 (2015).

[12] Y. Feng, R. Jaramillo, A. Banerjee, J. M. Honig, and T. F. Rosenbaum, Magnetism, Structure, and Charge Correlation at a Pressure-Induced Mott-Hubbard Insulator-Metal Transition, Phys. Rev. B 83, 035106 (2011).

[13] Y. Zhou, K. Kanoda, and T.-K. Ng, Quantum Spin Liquid States, Rev. Mod. Phys. 89, 025003 (2017).

[14] D. B. McWhan, A. Menth, J. P. Remeika, W. F. Brinkman, and T. M. Rice, Metal-Insulator Transitions in Pure and Doped $\mathrm{V}_{2} \mathrm{O}_{3}$, Phys. Rev. B 7, 1920 (1973).

[15] R. M. Moon, Antiferromagnetism in $\mathrm{V}_{2} \mathrm{O}_{3}$, Phys. Rev. Lett. 25, 527 (1970).

[16] D. B. McWhan, T. M. Rice, and J.P. Remeika, Mott Transition in Cr-Doped $\mathrm{V}_{2} \mathrm{O}_{3}$, Phys. Rev. Lett. 23, 1384 (1969).

[17] D. B. McWhan and J. P. Remeika, Metal-Insulator Transition in $\left(\mathrm{V}_{1-x} \mathrm{Cr}_{x}\right)_{2} \mathrm{O}_{3}$, Phys. Rev. B 2, 3734 (1970).

[18] Y. Ueda, K. Kosuge, and S. Kachi, Phase Diagram and Some Physical Properties of $\mathrm{V}_{2} \mathrm{O}_{3+x} \quad(0 \leq x \leq 0.080)$, J. Solid State Chem. 31, 171 (1980).

[19] H. Kuwamoto, J. M. Honig, and J. Appel, Electrical Properties of the $\left(\mathrm{V}_{1-x} \mathrm{Cr}_{x}\right)_{2} \mathrm{O}_{3}$ System, Phys. Rev. B 22, 2626 (1980).

[20] S. A. Carter, T. F. Rosenbaum, J. M. Honig, and J. Spalek, New Phase Boundary in Highly Correlated, Barely Metallic $\mathrm{V}_{2} \mathrm{O}_{3}$, Phys. Rev. Lett. 67, 3440 (1991).

[21] M. Yethiraj, Pure and Doped Vanadium Sesquioxide: A Brief Experimental Review, J. Solid State Chem. 88, 53 (1990).

[22] P. Limelette, A. Georges, D. Jérome, P. Wzietek, P. Metcalf, and J. M. Honig, Universality and Critical Behavior at the Mott Transition, Science 302, 89 (2003). 
[23] D. Grieger and M. Fabrizio, Low-Temperature Magnetic Ordering and Structural Distortions in Vanadium Sesquioxide $\mathrm{V}_{2} \mathrm{O}_{3}$, Phys. Rev. B 92, 075121 (2015).

[24] C. Castellani, C. R. Natoli, and J. Ranninger, Magnetic Structure of $\mathrm{V}_{2} \mathrm{O}_{3}$ in the Insulating Phase, Phys. Rev. B 18, 4945 (1978).

[25] C. Castellani, C. R. Natoli, and J. Ranninger, Insulating Phase of $\mathrm{V}_{2} \mathrm{O}_{3}$ : An Attempt at a Realistic Calculation, Phys. Rev. B 18, 4967 (1978).

[26] C. Castellani, C. R. Natoli, and J. Ranninger, Metal-Insulator Transition in Pure and Cr-Doped $\mathrm{V}_{2} \mathrm{O}_{3}$, Phys. Rev. B 18, 5001 (1978).

[27] M. J. Rozenberg, G. Kotliar, H. Kajueter, G. A. Thomas, D. H. Rapkine, J. M. Honig, and P. Metcalf, Optical Conductivity in Mott-Hubbard Systems, Phys. Rev. Lett. 75, 105 (1995).

[28] W. Bao, C. Broholm, S. A. Carter, T. F. Rosenbaum, G. Aeppli, S. F. Trevino, P. Metcalf, J. M. Honig, and J. Spalek, Incommensurate Spin Density Wave in Metallic $\mathrm{V}_{2-y} \mathrm{O}_{3}$, Phys. Rev. Lett. 71, 766 (1993).

[29] H. Kadowaki, K. Motoya, T. J. Sato, J. W. Lynn, J. A. Fernandez-Baca, and J. Kikuchi, Quantum Phase Transition in the Itinerant Antiferromagnet $\left(\mathrm{V}_{0.9} \mathrm{Ti}_{0.1}\right)_{2} \mathrm{O}_{3}$, Phys. Rev. Lett. 101, 096406 (2008).

[30] G. Aeppli, W. Bao, C. Broholm, S.-W. Cheong, P. Dai, S. M. Hayden, T. E. Mason, H. A. Mook, T. G. Perring et al., Magnetic Correlations in Doped Transition-Metal Oxides, in Spectroscopy of Mott Insulators and Correlated Metals, Springer Series in Solid-State Sciences, edited by A. Fujimori and Y. Tokura (Springer-Verlag, Berlin, 1995), Vol. 119, p. 205.

[31] W. Bao, C. Broholm, G. Aeppli, P. Dai, J. M. Honig, and P. Metcalf, Dramatic Switching of Magnetic Exchange in a Classic Transition Metal Oxide: Evidence for Orbital Ordering, Phys. Rev. Lett. 78, 507 (1997).

[32] T. M. Rice, Orbital-Spin Coupling in $\mathrm{V}_{2} \mathrm{O}_{3}$ and Related Oxides, in Spectroscopy of Mott Insulators and Correlated Metals (Ref. [30]), p. 221.

[33] Y. Q. Li, M. Ma, D. N. Shi, and F. C. Zhang, SU(4) Theory for Spin Systems with Orbital Degeneracy, Phys. Rev. Lett. 81, 3527 (1998).

[34] K. I. Kugel, D. I. Khomskii, A. O. Sboychakov, and S. V. Streltsov, Spin-Orbital Interaction for Face-Sharing Octahedra: Realization of a Highly Symmetric SU(4) Model, Phys. Rev. B 91, 155125 (2015).

[35] A. Joshi, M. Ma, F. Mila, D. N. Shi, and F. C. Zhang, Elementary Excitations in Magnetically Ordered Systems with Orbital Degeneracy, Phys. Rev. B 60, 6584 (1999).

[36] L. Paolasini, C. Vettier, F. de Bergevin, F. Yakhou, D. Mannix, A. Stunault, W. Neubeck, M. Altarelli, M. Fabrizio, P. A. Metcalf, and J. M. Honig, Orbital Occupancy Order in $\mathrm{V}_{2} \mathrm{O}_{3}$ : Resonant X-Ray Scattering Results, Phys. Rev. Lett. 82, 4719 (1999).

[37] M. Fabrizio, M. Altarelli, and M. Benfatto, X-Ray Resonant Scattering as a Direct Probe of Orbital Ordering in Transition-Metal Oxides, Phys. Rev. Lett. 80, 3400 (1998).

[38] S. Di Matteo, N. B. Perkins, and C. R. Natoli, Spin-1 Effective Hamiltonian with Three Degenerate Orbitals:
An Application to the Case of $\mathrm{V}_{2} \mathrm{O}_{3}$, Phys. Rev. B 65, 054413 (2002).

[39] J.-H. Park, L. H. Tjeng, A. Tanaka, J. W. Allen, C. T. Chen, P. Metcalf, J. M. Honig, F. M. F. de Groot, and G. A. Sawatzky, Spin and Orbital Occupation and Phase Transitions in $\mathrm{V}_{2} \mathrm{O}_{3}$, Phys. Rev. B 61, 11506 (2000).

[40] S. Yu. Ezhov, V. I. Anisimov, D. I. Khomskii, and G. A. Sawatzky, Orbital Occupation, Local Spin, and Exchange Interactions in $\mathrm{V}_{2} \mathrm{O}_{3}$, Phys. Rev. Lett. 83, 4136 (1999).

[41] K. Held, G. Keller, V. Eyert, D. Vollhardt, and V. I. Anisimov, Mott-Hubbard Metal-Insulator Transition in Paramagnetic $\mathrm{V}_{2} \mathrm{O}_{3}:$ An $\mathrm{LDA}+\operatorname{DMFT}(\mathrm{QMC})$ Study, Phys. Rev. Lett. 86, 5345 (2001).

[42] P. Hansmann, A. Toschi, G. Sangiovanni, T. Saha-Dasgupta, S. Lupi, M. Marsi, and K. Held, Mott-Hubbard Transition in $\mathrm{V}_{2} \mathrm{O}_{3}$ Revisited, Phys. Status Solidi B 250, 1251 (2013).

[43] F. Mila, R. Shiina, F.-C. Zhang, A. Joshi, M. Ma, V. Anisimov, and T.M. Rice, Orbitally Degenerate Spin-1 Model for Insulating $\mathrm{V}_{2} \mathrm{O}_{3}$, Phys. Rev. Lett. 85, 1714 (2000).

[44] R. Shiina, F. Mila, F.-C. Zhang, and T. M. Rice, Atomic Spin, Molecular Orbitals, and Anomalous Antiferromagnetism in Insulating $\mathrm{V}_{2} \mathrm{O}_{3}$, Phys. Rev. B 63, 144422 (2001).

[45] A. Joshi, M. Ma, and F. C. Zhang, Theory for Phase Transitions in Insulating $\mathrm{V}_{2} \mathrm{O}_{3}$, Phys. Rev. Lett. 86, 5743 (2001).

[46] L. F. Feiner, A. M. Oleś, and J. Zaanen, Quantum Melting of Magnetic Order due to Orbital Fluctuations, Phys. Rev. Lett. 78, 2799 (1997).

[47] I. Lo Vecchio, J. D. Denlinger, O. Krupin, B. J. Kim, P. A. Metcalf, S. Lupi, J. W. Allen, and A. Lanzara, Fermi Surface of Metallic $\mathrm{V}_{2} \mathrm{O}_{3}$ from Angle-Resolved Photoemission: Mid-Level Filling of $e_{g}^{\pi}$ Bands, Phys. Rev. Lett. 117, 166401 (2016).

[48] D. Grieger and F. Lechermann, Effect of Chromium Doping on the Correlated Electronic Structure of $\mathrm{V}_{2} \mathrm{O}_{3}$, Phys. Rev. B 90, 115115 (2014).

[49] X. Deng, A. Sternbach, K. Haule, D. N. Basov, and G. Kotliar, Shining Light on Transition-Metal Oxides: Unveiling the Hidden Fermi Liquid, Phys. Rev. Lett. 113, 246404 (2014).

[50] R. E. Word, S. A. Werner, W. B. Yelon, J. M. Honig, and S. Shivashankar, Spin Waves in Vanadium Sesquioxide $\mathrm{V}_{2} \mathrm{O}_{3}$, Phys. Rev. B 23, 3533 (1981).

[51] M. Yethiraj, S. A. Werner, W. B. Yelon, and J. M. Honig, Phonon Anomalies and the Magnetic Transition in Pure and Cr-Doped $\mathrm{V}_{2} \mathrm{O}_{3}$, Phys. Rev. B 36, 8675 (1987).

[52] W. Bao, C. Broholm, G. Aeppli, S. A. Carter, P. Dai, T. F. Rosenbaum, J. M. Honig, P. Metcalf, and S. F. Trevino, Magnetic Correlations and Quantum Criticality in the Insulating Antiferromagnetic, Insulating Spin Liquid, Renormalized Fermi Liquid, and Metallic Antiferromagnetic Phases of the Mott System $\mathrm{V}_{2} \mathrm{O}_{3}$, Phys. Rev. B 58, 12727 (1998).

[53] H. Harrison, R. Aragon, and C. Sandberg, Single Crystal Growth of the Transition Metal Monoxides by Skull Melting, Mater. Res. Bull. 15, 571 (1980). 
[54] P. D. Dernier and M. Marezio, Crystal Structure of the LowTemperature Antiferromagnetic Phase of $\mathrm{V}_{2} \mathrm{O}_{3}$, Phys. Rev. B 2, 3771 (1970).

[55] A. Jayaraman, D. B. McWhan, J. P. Remeika, and P. D. Dernier, Critical Behavior of the Mott Transition in Cr-Doped $\mathrm{V}_{2} \mathrm{O}_{3}$, Phys. Rev. B 2, 3751 (1970).

[56] G. E. Granroth, A. I. Kolesnikov, T. E. Sherline, J. P. Clancy, K. A. Ross, J. P. C. Ruff, B. D. Gaulin, and S. E. Nagler, SEQUOIA: A Newly Operating Chopper Spectrometer at the SNS, J. Phys. Conf. Ser. 251, 012058 (2010).

[57] M. B. Stone, J. L. Niedziela, D. L. Abernathy, L. DeBeerSchmitt, G. Ehlers, O. Garlea, G. E. Granroth, M. Graves-Brook, A. I. Kolesnikov, A. Podlesnyak, and B. Winn, A Comparison of Four Direct Geometry Time-ofFlight Spectrometers at the Spallation Neutron Source, Rev. Sci. Instrum. 85, 045113 (2014).

[58] O. Arnold, J.-C. Bilheux, J. M. Borreguero, A. Buts, S. I. Campbell, L. Chapon, M. Doucet, N. Draper, R. Ferraz Leal, M. A. Gigg et al., Mantid-Data Analysis and Visualization Package for Neutron Scattering and $\mu \mathrm{SR}$ Experiments, Nucl. Instrum. Methods Phys. Res., Sect. A 764, 156 (2014).

[59] S. Toth and B. Lake, Linear Spin Wave Theory for Single- $Q$ Incommensurate Magnetic Structures, J. Phys. Condens. Matter 27, 166002 (2015).

[60] P. Rozier, A. Ratuszna, and J. Galy, Comparative Structural and Electrical Studies of $\mathrm{V}_{2} \mathrm{O}_{3}$ and $\mathrm{V}_{2-x} \mathrm{Ni}_{x} \mathrm{O}_{3}(0<x<$ 0.75) Solid Solution, Z. Anorg. Allg. Chem. 628, 1236 (2002).

[61] K. Koepernik and H. Eschrig, Full-Potential Nonorthogonal Local-Orbital Minimum-Basis Band-Structure Scheme, Phys. Rev. B 59, 1743 (1999).

[62] J. P. Perdew, K. Burke, and M. Ernzerhof, Generalized Gradient Approximation Made Simple, Phys. Rev. Lett. 77, 3865 (1996).

[63] H. O. Jeschke, F. Salvat-Pujol, and R. Valentí, FirstPrinciples Determination of Heisenberg Hamiltonian Parameters for the Spin-1/2 Kagome Antiferromagnet $\mathrm{ZnCu}_{3}(\mathrm{OH})_{6} \mathrm{Cl}_{2}$, Phys. Rev. B 88, 075106 (2013).

[64] T. Saha-Dasgupta, O. K. Andersen, J. Nuss, A. I. Poteryaev, A. Georges, and A. I. Lichtenstein, Electronic Structure of $\mathrm{V}_{2} \mathrm{O}_{3}$ : Wannier Orbitals from LDA-NMTO Calculations, arXiv:0907.2841.

[65] D. Khomskii, Transition Metal Compounds (Cambridge University Press, Cambridge, England, 2014).

[66] G. Khaliullin, Orbital Order and Fluctuations in Mott Insulators, Prog. Theor. Phys. Suppl. 160, 155 (2005).

[67] A. Menth and J.P. Remeika, Magnetic Properties of $\left(\mathrm{V}_{1-x} \mathrm{Cr}_{x}\right)_{2} \mathrm{O}_{3}$, Phys. Rev. B 2, 3756 (1970).

[68] A. F. Albuquerque, D. Schwandt, B. Hetényi, S. Capponi, M. Mambrini, and A. M. Läuchli, Phase Diagram of a Frustrated Quantum Antiferromagnet on the Honeycomb Lattice: Magnetic Order versus Valence-Bond Crystal Formation, Phys. Rev. B 84, 024406 (2011).

[69] P. H. Conlon and J. T. Chalker, Absent Pinch Points and Emergent Clusters: Further Neighbor Interactions in the Pyrochlore Heisenberg Antiferromagnet, Phys. Rev. B 81, 224413 (2010).

[70] H. E. Stanley, Spherical Model as the Limit of Infinite Spin Dimensionality, Phys. Rev. 176, 718 (1968).
[71] F. Mezzacapo and M. Boninsegni, Ground-State Phase Diagram of the Quantum $J_{1}-J_{2}$ Model on the Honeycomb Lattice, Phys. Rev. B 85, 060402 (2012).

[72] S.-H. Lee, C. Broholm, G. Aeppli, A. P. Ramirez, T. G. Perring, C. J. Carlile, M. Adams, T. J. L. Jones, and B. Hessen, Spin-Glass and Non-Spin-Glass Features of a Geometrically Frustrated Magnet, Europhys. Lett. 35, 127 (1996).

[73] E. J. Samuelsen, M. T. Hutchings, and G. Shirane, Inelastic Neutron Scattering Investigation of Spin Waves and Magnetic Interactions in $\mathrm{Cr}_{2} \mathrm{O}_{3}$, Physica (Amsterdam) 48, 13 (1970).

[74] M. M. Seikh, C. Narayana, A. K. Sood, P. Murugavel, M. W. Kim, P. A. Metcalf, J. M. Honig, and C. N. R. Rao, A Brillouin Study of the Temperature Dependence of the Acoustic Modes across the Insulator-Metal Transitions in $\mathrm{V}_{2} \mathrm{O}_{3}$ and $C r$-Doped $\mathrm{V}_{2} \mathrm{O}_{3}$, Solid State Commun. 138, 466 (2006).

[75] L. Dillemans, R. R. Lieten, M. Menghini, T. Smets, J. W. Seo, and J.-P. Locquet, Correlation between Strain and the Metal-Insulator Transition in Epitaxial $\mathrm{V}_{2} \mathrm{O}_{3}$ Thin Films Grown by Molecular Beam Epitaxy, Thin Solid Films 520, 4730 (2012).

[76] S. Yonezawa, Y. Muraoka, Y. Ueda, and Z. Hiroi, Epitaxial Strain Effects on the Metal-Insulator Transition in $\mathrm{V}_{2} \mathrm{O}_{3}$ Thin Films, Solid State Commun. 129, 245 (2004).

[77] J. de la Venta, S. Wang, J. G. Ramirez, and I. K. Schuller, Control of Magnetism across Metal to Insulator Transitions, Appl. Phys. Lett. 102, 122404 (2013).

[78] J. de la Venta, S. Wang, T. Saerbeck, J. G. Ramirez, I. Valmianski, and I. K. Schuller, Coercivity Enhancement in $\mathrm{V}_{2} \mathrm{O}_{3} / \mathrm{Ni}$ Bilayers Driven by Nanoscale Phase Coexistence, Appl. Phys. Lett. 104, 062410 (2014).

[79] M. Matsuura, Y. Endoh, H. Hiraka, K. Yamada, A. S. Mishchenko, N. Nagaosa, and I. V. Solovyev, Classical and Quantum Spin Dynamics in the FCC Antiferromagnet $\mathrm{NiS}_{2}$ with Frustration, Phys. Rev. B 68, 094409 (2003).

[80] H.-S. Kim, J. Im, M. J. Han, and H. Jin, Spin-Orbital Entangled Molecular $J_{\text {eff }}$ States in Lacunar Spinel Compounds, Nat. Commun. 5, 3988 (2014).

[81] M. Y. Jeong, S. H. Chang, B. H. Kim, J.-H. Sim, A. Said, D. Casa, T. Gog, E. Janod, L. Cario, S. Yunoki et al., Direct Experimental Observation of the Molecular $J_{\text {eff }}=3 / 2$ Ground State in the Lacunar Spinel $\mathrm{GaTa}_{4} \mathrm{Se}_{8}$, Nat. Commun. 8, 782 (2017).

[82] Y. Okamoto, M. Nohara, H. Aruga-Katori, and H. Takagi, Spin-Liquid State in the $S=1 / 2$ Hyperkagome Antiferromagnet $\mathrm{Na}_{4} \mathrm{Ir}_{3} \mathrm{O}_{8}$, Phys. Rev. Lett. 99, 137207 (2007).

[83] D. Podolsky, A. Paramekanti, Y. B. Kim, and T. Senthil, Mott Transition between a Spin-Liquid Insulator and a Metal in Three Dimensions, Phys. Rev. Lett. 102, 186401 (2009).

[84] T. Senthil, Theory of a Continuous Mott Transition in Two Dimensions, Phys. Rev. B 78, 045109 (2008).

[85] L. Savary and L. Balents, Quantum Spin Liquids: A Review, Rep. Prog. Phys. 80, 016502 (2017).

[86] A. Pustogow, M. Bories, A. Löhle, R. Rösslhuber, E. Zhukova, B. Gorshunov, S. Tomić, J. A. Schlueter, R. Hübner, T. Hiramatsu et al., Quantum Spin Liquids Unveil the Genuine Mott State, Nat. Mater. 17, 773 (2018). 
[87] M. Kresch, M. Lucas, O. Delaire, J. Y. Y. Lin, and B. Fultz, Phonons in Aluminum at High Temperatures Studied by Inelastic Neutron Scattering, Phys. Rev. B 77, 024301 (2008).

[88] E. R. Ylvisaker, W. E. Pickett, and K. Koepernik, Anisotropy and Magnetism in the LSDA + U Method, Phys. Rev. B 79, 035103 (2009).

[89] T. Mizokawa and A. Fujimori, Electronic Structure and Orbital Ordering in Perovskite-Type 3d Transition-Metal
Oxides Studied by Hartree-Fock Band-Structure Calculations, Phys. Rev. B 54, 5368 (1996).

[90] J. Y. Y. Lin, H. L. Smith, G. E. Granroth, D. L. Abernathy, M. D. Lumsden, B. Winn, A. A. Aczel, M. Aivazis, and B. Fultz, MCVINE: An Object Oriented Monte Carlo Neutron Ray Tracing Simulation Package, Nucl. Instrum. Methods Phys. Res., Sect. A 810, 86 (2016).

[91] M. Könneckeet al., The NeXus Data Format, J. Appl. Crystallogr. 48, 301 (2015). 